\title{
Convergence rates for the numerical approximation of the 2D stochastic Navier-Stokes equations
}

\author{
Dominic Breit ${ }^{1} \cdot$ Alan Dodgson $^{1}$
}

Received: 2 October 2019 / Revised: 10 August 2020 / Accepted: 22 November 2020 /

Published online: 11 February 2021

(c) The Author(s) 2021

\begin{abstract}
We study stochastic Navier-Stokes equations in two dimensions with respect to periodic boundary conditions. The equations are perturbed by a nonlinear multiplicative stochastic forcing with linear growth (in the velocity) driven by a cylindrical Wiener process. We establish convergence rates for a finite-element based space-time approximation with respect to convergence in probability (where the error is measured in the $L_{t}^{\infty} L_{x}^{2} \cap L_{t}^{2} W_{x}^{1,2}$-norm). Our main result provides linear convergence in space and convergence of order (almost) $1 / 2$ in time. This improves earlier results from Carelli and Prohl (SIAM J Numer Anal 50(5):2467-2496, 2012) where the convergence rate in time is only (almost) $1 / 4$. Our approach is based on a careful analysis of the pressure function using a stochastic pressure decomposition.
\end{abstract}

Mathematics Subject Classification $65 \mathrm{M} 15 \cdot 65 \mathrm{C} 30 \cdot 60 \mathrm{H} 15 \cdot 60 \mathrm{H} 35$

\section{Introduction}

In this paper we are concerned with the stochastic Navier-Stokes equations

$$
\left\{\begin{array}{lr}
\operatorname{du}=\mu \Delta \mathbf{u} \mathrm{d} t-(\nabla \mathbf{u}) \mathbf{u} \mathrm{d} t-\nabla \pi \mathrm{d} t+\Phi(\mathbf{u}) \mathrm{d} W & \text { in } Q, \\
\operatorname{div} \mathbf{u}=0 & \text { in } Q, \\
\mathbf{u}(0)=\mathbf{u}_{0} & \text { in } \mathcal{O},
\end{array}\right.
$$

on a filtered probability space $\left(\Omega, \mathfrak{F},\left(\mathfrak{F}_{t}\right)_{t \geq 0}, \mathbb{P}\right)$. The equations are perturbed by an $\left(\mathfrak{F}_{t}\right)$-Wiener process (possibly infinite dimensional) and $\Phi$ grows linearly in u (see Sect. 2.1 for the precise assumptions). The quantity $\mu>0$ is the viscosity of the fluid

$\triangle$ Dominic Breit

d.breit@hw.ac.uk

Alan Dodgson

ad335@hw.ac.uk

1 Department of Mathematics, Heriot-Watt University, Riccarton, Edinburgh EH14 4AS, UK 
and $\mathbf{u}_{0}$ is a given (random) initial datum. Here the unknowns are the velocity field $\mathbf{u}: \Omega \times Q \rightarrow \mathbb{R}^{N}$ and the pressure $\pi: \Omega \times Q \rightarrow \mathbb{R}$, where $Q=(0, T) \times \mathcal{O}$ and $\mathcal{O} \subset \mathbb{R}^{N}$ with $N=2,3$.

The stochastic perturbation in the balance of momentum (1.1) 1 can take into account for physical, empirical or numerical uncertainties and thermodynamical fluctuations. In addition to that, a main reason why the stochastic Navier-Stokes equations (1.1) became so popular in fluid mechanical research is their application to turbulence theory (see, for instance, [2,21]). Its mathematical investigation started in the 70's with the pioneering paper of Bensoussan and Temam [1]. They provide a semi-deterministic approach based on the flow-transformation. A first fully stochastic theory has been developed by Flandoli and Gatarek [14] by showing the existence of a martingale solution. These solutions are weak in the stochastic sense meaning that the underlying probability space as well the Wiener process $W$ are not a priori known but become an integral part of the solution. In two dimensions, when uniqueness is known, a stochastically strong solution exists (it is defined on a given probability space with a given Wiener process), see [12]. Nowadays there is a huge amount of literature concerning the analysis of (1.1) and most of the results from the deterministic theory found their stochastic counterpart. For an overview we refer to the recent survey article [25].

The situation about the numerical approximation of (1.1) is totally different and only very few results are available. In [8] a fully practical space-time approximation for the three-dimensional stochastic Navier-Stokes equations in a bounded domain is studied. It is shown that the sequence of approximate solutions converges in law (up to a subsequence) to a martingale solution if both discretization parameters tend to zero. This is the best result one can hope for without using some unproven hypotheses about the space-regularity of solutions (or to be content with local-in-time results). In two dimensions the situation is much better, at least if periodic boundary conditions are considered, that is

$$
\mathcal{O}=\mathbb{T}^{2}=\left(\left.(-\pi, \pi)\right|_{\{-\pi, \pi\}}\right)^{2}
$$

The space-regularity of the unique strong solution is well-known (see for instance [20]). Based on this the convergence rates for a finite-element based space-time approximation is analysed in [9]. The result is linear convergence in space and convergence of order (almost) 1/4 in time. The precise estimate comparing the solution $\mathbf{u}$ and its space-time approximation $\mathbf{u}_{h, m}$ reads as

$$
\begin{gathered}
\mathbb{E}\left[\mathbf { 1 } _ { \Omega _ { \Delta t , h } } \left(\max _{1 \leq m \leq M}\left\|\mathbf{u}\left(t_{m}\right)-\mathbf{u}_{h, m}\right\|_{L_{x}^{2}}^{2}\right.\right. \\
\left.\left.+\sum_{m=1}^{M} \Delta t\left\|\nabla \mathbf{u}\left(t_{m}\right)-\nabla \mathbf{u}_{h, m}\right\|_{L_{x}^{2}}^{2}\right)\right] \\
\leq c\left(h^{2}+(\Delta t)^{2 \alpha}\right)
\end{gathered}
$$

for any $\alpha<1 / 4$. Here we have $\Omega_{\Delta t, h} \subset \Omega$ with $\mathbb{P}\left(\Omega \backslash \Omega_{\Delta t, h}\right) \rightarrow 0$ as $\Delta t, h \rightarrow 0$. Consequently, one can infer from (1.2) convergence in probability (as defined by Printems [24]) with asymptotic rates (almost) $1 / 4$ and 1 respectively. The aim of 
the present paper is to improve the convergence rates in time from (almost) $1 / 4$ to (almost) $1 / 2$, see Theorem 3 for the precise statement. This is certainly the optimal convergence rate in time in view of the stochastic forcing and should also be considered as the natural result because of the space-regularity of the solution.

The reason for the low convergence rate in time in [9] is the low time-regularity of the pressure function $\pi$ in (1.1). Its appearance can only be avoided when working with finite element functions which are exactly divergence-free. Unfortunately, their construction is quite complicated such that the preferred descretizations (such as the Taylor-Hood, the Crouzeix-Raviart, and the MINI element, see [7,16,17]) are only asymptotically divergence-free. The low regularity of the pressure gradient arises from the stochastic forcing (in fact, $\nabla \pi$ behaves as $\mathrm{d} W$ ) and improvements do not seem possible unless the noise is divergence-free (this is quite restrictive as it only allows certain additive or linear multiplicative noise). In the general case (non-divergencefree finite elements and nonlinear multiplicative noise) a more subtle analysis of the pressure function is required. Our main idea is a decomposition of the pressure into a deterministic and a stochastic component (such a decomposition first appeared in [5]). The deterministic pressure part behaves as the convective term $\mathbf{u} \otimes \mathbf{u}$. The latter one can be estimated along the lines of [9] (following classical deterministic arguments combined with a discrete stopping time). In addition a second stochastic integral appears which behaves similarly to the original stochastic integral. Although the timeregularity of this part has not improved, we benefit from the averaging properties of the Itô-integral. Combining these ideas finally leads to the optimal convergence rate in Theorem 3.

The paper is organized as follows. In Sect. 2 we present the mathematical framework, that is the probability setup, the concept of solutions and their qualitative properties. In particular, we give improved (compared to [9]) results on the timeregularity of $\nabla \mathbf{u}$, see Corollary 2 b). This is needed in Sect. 3 in order to estimate the error between the continuous solution and the time-discrete solution. The heart of the paper is Sect. 4 were we estimate the error between the time-discrete solution and the space-time discretization. Crucial tools are the space-regularity of the time-discrete solution from [8] and the decomposition of the corresponding pressure function.

\section{Mathematical framework}

\subsection{Probability setup}

Let $\left(\Omega, \mathfrak{F},\left(\mathfrak{F}_{t}\right)_{t \geq 0}, \mathbb{P}\right)$ be a stochastic basis with a complete, right-continuous filtration. The process $W$ is a cylindrical Wiener process, that is, $W(t)=\sum_{k \geq 1} \beta_{k}(t) e_{k}$ with $\left(\beta_{k}\right)_{k \geq 1}$ being mutually independent real-valued standard Wiener processes relative to $\left(\mathfrak{F}_{t}\right)$ and $\left(e_{k}\right)_{k \geq 1}$ is a complete orthonormal system in a separable Hilbert space $\mathfrak{U}$. To give the precise definition of the diffusion coefficient $\Phi$, consider $\mathbf{z} \in L^{2}\left(\mathbb{T}^{2}\right)$ and let $\Phi(\mathbf{z}): \mathfrak{U} \rightarrow L^{2}\left(\mathbb{T}^{2}\right)$ be defined by $\Phi(\mathbf{z}) e_{k}=\mathbf{g}_{k}(\cdot, \mathbf{z}(\cdot))$. In particular, we suppose that $\mathbf{g}_{k} \in C^{1}\left(\mathbb{T}^{2} \times \mathbb{R}^{2}\right)$ and that the following conditions hold 


$$
\begin{gathered}
\sum_{k \geq 1}\left|\mathbf{g}_{k}(x, \boldsymbol{\xi})\right|^{2} \leq c\left(1+|\boldsymbol{\xi}|^{2}\right), \quad \sum_{k \geq 1}\left|\nabla_{\boldsymbol{\xi}} \mathbf{g}_{k}(x, \boldsymbol{\xi})\right|^{2} \leq c \\
\sum_{k \geq 1}\left|\nabla_{x} \mathbf{g}_{k}(x, \boldsymbol{\xi})\right|^{2} \leq c\left(1+|\boldsymbol{\xi}|^{2}\right), \quad x \in \mathbb{T}^{2}, \boldsymbol{\xi} \in \mathbb{R}^{2} .
\end{gathered}
$$

If we are interested in higher regularity some further assumptions are in place and we require additionally $\mathbf{g}_{k} \in C^{2}\left(\mathbb{T}^{2} \times \mathbb{R}^{2}\right)$ together with

$$
\begin{gathered}
\sum_{k \geq 1}\left|\nabla_{x}^{2} \mathbf{g}_{k}(x, \boldsymbol{\xi})\right|^{2} \leq c\left(1+|\boldsymbol{\xi}|^{2}\right), \quad \sum_{k \geq 1}\left|\nabla_{\xi}^{2} \mathbf{g}_{k}(x, \boldsymbol{\xi})\right|^{2} \leq \frac{c}{1+|\boldsymbol{\xi}|^{2}} \\
\sum_{k \geq 1}\left|\nabla_{x} \nabla_{\boldsymbol{\xi}} \mathbf{g}_{k}(x, \boldsymbol{\xi})\right|^{2} \leq c, \quad x \in \mathbb{T}^{2}, \boldsymbol{\xi} \in \mathbb{R}^{2}
\end{gathered}
$$

We remark that the first inequality of (2.1) implies

$$
\|\Phi(\mathbf{u})\|_{L_{2}\left(\mathfrak{U} ; L_{x}^{2}\right)} \leq c\left(1+\|\mathbf{u}\|_{L_{x}^{2}}\right) \quad \forall \mathbf{u} \in L^{2}\left(\mathbb{T}^{2}\right),
$$

all inequalities from (2.1) imply

$$
\|\Phi(\mathbf{u})\|_{L_{2}\left(\mathfrak{U} ; W_{x}^{1,2}\right)} \leq c\left(1+\|\mathbf{u}\|_{\left.W_{x}^{1,2}\right)} \forall \mathbf{u} \in W^{1,2}\left(\mathbb{T}^{2}\right),\right.
$$

and (2.1) finally yields

$$
\|\Phi(\mathbf{u})\|_{L_{2}\left(\mathfrak{U} ; W_{x}^{2,2}\right)} \leq c\left(1+\|\mathbf{u}\|_{W_{x}^{2,2}}\right) \quad \forall \mathbf{u} \in W^{2,2}\left(\mathbb{T}^{2}\right) .
$$

In fact, all our results apply if we replace (2.1) and (2.2) by the corresponding norm estimates above.

Furthermore, the conditions imposed on $\Phi$, particularly the first assumption from (2.1), allow us to define stochastic integrals Given an $\left(\mathfrak{F}_{t}\right)$-progressively measurable process $\mathbf{u} \in L^{2}\left(\Omega ; L^{2}\left(0, T ; L^{2}\left(\mathbb{T}^{2}\right)\right)\right)$, the stochastic integral

$$
t \mapsto \int_{0}^{t} \Phi(\mathbf{u}) \mathrm{d} W
$$

is a well defined process taking values in $L^{2}\left(\mathbb{T}^{2}\right)$ (see [13] for the detailed construction). Moreover, we can multiply by test functions to obtain

$$
\left\langle\int_{0}^{t} \Phi(\mathbf{u}) \mathrm{d} W, \boldsymbol{\phi}\right\rangle=\sum_{k \geq 1} \int_{0}^{t}\left\langle\mathbf{g}_{k}(\mathbf{u}), \boldsymbol{\phi}\right\rangle \mathrm{d} \beta_{k}, \quad \boldsymbol{\phi} \in L^{2}\left(\mathbb{T}^{2}\right) .
$$

Similarly, we can define stochastic integrals with values in $W^{1,2}\left(\mathbb{T}^{2}\right)$ and $W^{2,2}\left(\mathbb{T}^{2}\right)$ respectively if $\mathbf{u}$ belongs to the corresponding class.

The following lemma is a helpful tool to analysis the time-regularity of stochastic integrals (see, e.g., [6, Lemma 9.1.3. b)] or [19, Lemma 4.6]). 
Lemma 1 Let $\psi \in L^{r}\left(\Omega ; L^{r}\left(0, T ; L_{2}\left(\mathfrak{U}, L^{2}\left(\mathbb{T}^{2}\right)\right)\right)\right), r>2$, by an $\left(\mathfrak{F}_{t}\right)$-progressively measureable process and $W$ a cylindrical $\left(\mathfrak{F}_{t}\right)$-Wiener process on $\mathfrak{U}$. Then the paths of the process $Z_{t}:=\int_{0}^{t} \psi \mathrm{d} W$ are $\mathbb{P}$-a.s. Hölder continuous with exponent $\alpha \in\left(\frac{1}{r}, \frac{1}{2}\right)$ and it holds

$$
\mathbb{E}\left[\|Z\|_{C^{\alpha}\left([0, T] ; L^{2}\left(\mathbb{T}^{2}\right)\right)}^{r}\right] \leq c_{\alpha} \mathbb{E}\left[\int_{0}^{T}\|\psi\|_{L_{2}\left(\mathfrak{U}, L^{2}\left(\mathbb{T}^{2}\right)\right)}^{2} \mathrm{~d} t\right]^{\frac{r}{2}} .
$$

\subsection{The concept of solutions}

In dimension two, pathwise uniqueness for weak solutions is known under (2.1), we refer the reader for instance to Capiński-Cutland [12] and Capiński [11]. Consequently, we may work with the definition of a weak pathwise solution.

Definition 1 Let $\left(\Omega, \mathfrak{F},\left(\mathfrak{F}_{t}\right)_{t \geq 0}, \mathbb{P}\right)$ be a given stochastic basis with a complete rightcontinuous filtration and an $\left(\mathfrak{F}_{t}\right)$-cylindrical Wiener process $W$. Let $\mathbf{u}_{0}$ be an $\mathfrak{F}_{0^{-}}$ measurable random variable. Then $\mathbf{u}$ is called a weak pathwise solution to (1.1) with the initial condition $\mathbf{u}_{0}$ provided

(a) the velocity field $\mathbf{u}$ is $\left(\mathfrak{F}_{t}\right)$-adapted and

$$
\mathbf{u} \in C\left([0, T] ; L_{\text {div }}^{2}\left(\mathbb{T}^{2}\right)\right) \cap L^{2}\left(0, T ; W_{\text {div }}^{1,2}\left(\mathbb{T}^{2}\right)\right) \quad \mathbb{P} \text {-a.s. },
$$

(b) the momentum equation

$$
\begin{aligned}
\int_{\mathbb{T}^{2}} \mathbf{u}(t) \cdot \boldsymbol{\varphi} \mathrm{d} x-\int_{\mathbb{T}^{2}} \mathbf{u}_{0} \cdot \boldsymbol{\varphi} \mathrm{d} x \\
=\int_{0}^{t} \int_{\mathbb{T}^{2}} \mathbf{u} \otimes \mathbf{u}: \nabla \boldsymbol{\varphi} \mathrm{d} x \mathrm{~d} t-\mu \int_{0}^{t} \int_{\mathbb{T}^{2}} \nabla \mathbf{u}: \nabla \boldsymbol{\varphi} \mathrm{d} x \mathrm{~d} s \\
\quad+\int_{0}^{t} \int_{\mathbb{T}^{2}} \Phi(\mathbf{u}) \cdot \boldsymbol{\varphi} \mathrm{d} x \mathrm{~d} W .
\end{aligned}
$$

holds $\mathbb{P}$-a.s. for all $\varphi \in C_{\mathrm{div}}^{\infty}\left(\mathbb{T}^{2}\right)$ and all $t \in[0, T]$.

Theorem 1 Let $N=2$ and assume that $\Phi$ satisfies $(2.1)$. Let $\left(\Omega, \mathfrak{F},\left(\mathfrak{F}_{t}\right)_{t \geq 0}, \mathbb{P}\right)$ be a given stochastic basis with a complete right-continuous filtration and an $\left(\mathfrak{F}_{t}\right)$ cylindrical Wiener process $W$. Let $\mathbf{u}_{0}$ be an $\mathfrak{F}_{0}$-measurable random variable such that $\mathbf{u}_{0} \in L^{r}\left(\Omega ; L_{\mathrm{div}}^{2}\left(\mathbb{T}^{2}\right)\right)$ for some $r>2$. Then there exists a unique weak pathwise solution to (1.1) in the sense of Definition 1 with the initial condition $\mathbf{u}_{0}$.

Now, for $\phi \in C^{\infty}\left(\mathbb{T}^{2}\right)$ we can insert $\phi-\nabla \Delta^{-1} \operatorname{div} \phi$ and obtain

$$
\begin{aligned}
& \int_{\mathbb{T}^{2}} \mathbf{u}(t) \cdot \boldsymbol{\varphi} \mathrm{d} x+\int_{0}^{t} \int_{\mathbb{T}^{2}} \mu \nabla \mathbf{u}: \nabla \boldsymbol{\phi} \mathrm{d} x \mathrm{~d} \sigma-\int_{0}^{t} \int_{\mathbb{T}^{2}} \mathbf{u} \otimes \mathbf{u}: \nabla \boldsymbol{\phi} \mathrm{d} x \mathrm{~d} \sigma \\
& =\int_{\mathbb{T}^{2}} \mathbf{u}(0) \cdot \boldsymbol{\varphi} \mathrm{d} x+\int_{0}^{t} \int_{\mathbb{T}^{2}} \pi_{\operatorname{det}} \operatorname{div} \boldsymbol{\phi} \mathrm{d} x \mathrm{~d} \sigma
\end{aligned}
$$




$$
+\int_{\mathbb{T}^{2}} \int_{0}^{t} \Phi(\mathbf{u}) \mathrm{d} W \cdot \boldsymbol{\varphi} \mathrm{d} x+\int_{\mathbb{T}^{2}} \int_{0}^{t} \Phi^{\pi} \mathrm{d} W \cdot \boldsymbol{\varphi} \mathrm{d} x
$$

where

$$
\begin{aligned}
\pi_{\mathrm{det}} & =-\Delta^{-1} \operatorname{div} \operatorname{div}(\mathbf{u} \otimes \mathbf{u}), \\
\Phi^{\pi} & =-\nabla \Delta^{-1} \operatorname{div} \Phi(\mathbf{u}) .
\end{aligned}
$$

This corresponds to the stochastic pressure decomposition introduced in [5] (see also [6, Chap. 3] for a slightly different presentation). However, the situation with periodic boundary conditions we are considering here is much easier as the harmonic component of the pressure disappears.

\subsection{Regularity of solutions}

Lemma 2 Let the assumptions of Theorem 1 be satisfied.

(a) We have

$$
\mathbb{E}\left[\sup _{0 \leq t \leq T} \int_{\mathbb{T}^{2}}|\mathbf{u}|^{2} \mathrm{~d} x+\int_{Q}|\nabla \mathbf{u}|^{2} \mathrm{~d} x \mathrm{~d} t\right]^{\frac{r}{2}} \leq c_{r} \mathbb{E}\left[1+\left\|\mathbf{u}_{0}\right\|_{L_{x}^{2}}^{2}\right]^{\frac{r}{2}} .
$$

(b) Assume that $\mathbf{u}_{0} \in L^{r}\left(\Omega, W_{\text {div }}^{1,2}\left(\mathbb{T}^{2}\right)\right)$ for some $r \geq 2$. Then we have

$$
\mathbb{E}\left[\sup _{0 \leq t \leq T} \int_{\mathbb{T}^{2}}|\nabla \mathbf{u}|^{2} \mathrm{~d} x+\int_{Q}\left|\nabla^{2} \mathbf{u}\right|^{2} \mathrm{~d} x \mathrm{~d} t\right]^{\frac{r}{2}} \leq c_{r} \mathbb{E}\left[1+\left\|\mathbf{u}_{0}\right\|_{W_{x}^{1,2}}^{2}\right]^{\frac{r}{2}}
$$

(c) Assume that $\mathbf{u}_{0} \in L^{r}\left(\Omega, W_{\text {div }}^{2,2}\left(\mathbb{T}^{2}\right)\right) \cap L^{5 r}\left(\Omega, W_{\text {div }}^{1,2}\left(\mathbb{T}^{2}\right)\right)$ for some $r \geq 2$ and that (2.2) holds. Then we have

$$
\begin{aligned}
\mathbb{E}\left[\sup _{0 \leq t \leq T} \int_{\mathbb{T}^{2}}\left|\nabla^{2} \mathbf{u}\right|^{2} \mathrm{~d} x\right. & \left.+\int_{Q}\left|\nabla^{3} \mathbf{u}\right|^{2} \mathrm{~d} x \mathrm{~d} t\right]^{\frac{r}{2}} \\
& \leq c_{r} \mathbb{E}\left[1+\left\|\mathbf{u}_{0}\right\|_{W_{x}^{2,2}}^{2}+\left\|\mathbf{u}_{0}\right\|_{W_{x}^{1,2}}^{10}\right]^{\frac{r}{2}}
\end{aligned}
$$

Proof Part (a) is the standard a priori estimate which follows from applying Itô's formula to the functional $f(\mathbf{u})=\frac{1}{2}\|\mathbf{u}\|_{L_{x}^{2}}^{2}$ (and using Burkholder-Davis-Gundy inequality, assumption (2.1) and Gronwall's lemma). Note that this is legit in two dimensions since we have $\mathbf{u} \otimes \mathbf{u} \in L^{2}(Q) \mathbb{P}$-a.s. by Ladyshenskaya's inequality.

The proof of (b) and (c) is quite similar to [20, Corollary 2.4.13]. However, we are working with a different setup. So, we decided to give a formal proof although it is certainly known to experts. The proof can be made rigorous by working with a Galerkin-type approximation and show that the following estimates are uniform with 
respect to the dimension of the ansatz space. Such a procedure is quite standard, so we leave the details to the reader.

In order to show (b) we apply Itô's formula to the function $f_{\gamma}(\mathbf{u}):=\frac{1}{2}\left\|\partial_{\gamma} \mathbf{u}\right\|_{L_{x}^{2}}^{2}$ (with $\gamma \in\{1,2\}$ ) and obtain

$$
\begin{aligned}
\frac{1}{2}\left\|\partial_{\gamma} \mathbf{u}(t)\right\|_{L_{x}^{2}}^{2}= & \frac{1}{2}\left\|\partial_{\gamma} \mathbf{u}_{0}\right\|_{L_{x}^{2}}^{2}+\int_{0}^{t} f_{\gamma}^{\prime}(\mathbf{u}) \mathrm{d} \mathbf{u}+\frac{1}{2} \int_{0}^{t} f_{\gamma}^{\prime \prime}(\mathbf{u}) \mathrm{d}\langle\langle\mathbf{u}\rangle\rangle \\
= & \frac{1}{2}\left\|\partial_{\gamma} \mathbf{u}_{0}\right\|_{L_{x}^{2}}^{2}+\int_{\mathbb{T}^{2}} \int_{0}^{t} \partial_{\gamma} \mathbf{u} \cdot \mathrm{d} \partial_{\gamma} \mathbf{u} \mathrm{d} x \\
& +\frac{1}{2} \int_{\mathbb{T}^{2}} \int_{0}^{t} \mathrm{~d}\left\|\int_{0}^{\cdot} \partial_{\gamma}(\Phi(\mathbf{u}) \mathrm{d} W)\right\| \mathrm{d} x \\
= & :(I)+(I I)+(I I I) .
\end{aligned}
$$

We take the supremum in time, the $\frac{r}{2}$ th power and apply expectations. Summing over $\gamma$, we find

$$
\begin{aligned}
(I I) & =-(I I)_{1}-(I I)_{2}+(I I)_{3}, \\
(I I)_{1} & :=\mu \int_{0}^{t} \int_{\mathbb{T}^{2}}\left|\nabla^{2} \mathbf{u}\right|^{2} \mathrm{~d} x \mathrm{~d} \sigma \\
(I I)_{2} & :=\int_{\mathbb{T}^{2}} \int_{0}^{t} \partial_{\gamma} \mathbf{u} \cdot \partial_{\gamma}(\Phi(\mathbf{u}) \mathrm{d} W) \mathrm{d} x, \\
(I I)_{3} & :=\int_{0}^{t} \int_{\mathbb{T}^{2}}(\nabla \mathbf{u}) \mathbf{u} \cdot \Delta \mathbf{u} \mathrm{d} x \mathrm{~d} \sigma .
\end{aligned}
$$

In two dimensions we have $(I I)_{3}=0$ by elementary calculations. So we are left with estimating $(I I)_{2}$ and obtain

$$
\begin{aligned}
(I I)_{2}= & \sum_{k} \int_{\mathbb{T}^{2}} \int_{0}^{t} \partial_{\gamma} \mathbf{u} \cdot \partial_{\gamma}\left(\Phi(\mathbf{u}) e_{k} \mathrm{~d} \beta_{k}\right) \mathrm{d} x \\
= & \sum_{k} \int_{\mathbb{T}^{2}} \int_{0}^{t} \partial_{\gamma} \mathbf{u} \cdot \partial_{\gamma}\left(\mathbf{g}_{k}(\cdot, \mathbf{u}) \mathrm{d} \beta_{k}\right) \mathrm{d} x \\
= & \sum_{k} \int_{\mathbb{T}^{2}} \int_{0}^{t} \nabla_{\xi} \mathbf{g}_{k}(\cdot, \mathbf{u})\left(\partial_{\gamma} \mathbf{u}, \partial_{\gamma} \mathbf{u}\right) \mathrm{d} \beta_{k} \mathrm{~d} x \\
& +\sum_{k} \int_{\mathbb{T}^{2}} \int_{0}^{t} \partial_{\gamma} \mathbf{u} \cdot \partial_{\gamma} \mathbf{g}_{k}(\cdot, \mathbf{u}) \mathrm{d} \beta_{k}(\sigma) \mathrm{d} x \\
= & :(I I)_{2}^{1}+(I I)_{2}^{2} .
\end{aligned}
$$


On account of assumption (2.1), Burkholder-Davis-Gundy inequality and Young's inequality we obtain for arbitrary $\delta>0$

$$
\begin{aligned}
\mathbb{E}\left[\sup _{0 \leq t \leq T}\left|(I I)_{2}^{1}\right|\right]^{\frac{r}{2}} & \leq \mathbb{E}\left[\sup _{0 \leq t \leq T}\left|\int_{0}^{t} \sum_{k} \int_{\mathbb{T}^{2}} \nabla_{\xi} \mathbf{g}_{k}(\cdot, \mathbf{u})\left(\partial_{\gamma} \mathbf{u}, \partial_{\gamma} \mathbf{u}\right) \mathrm{d} x \mathrm{~d} \beta_{k}\right|\right]^{\frac{r}{2}} \\
& \leq c \mathbb{E}\left[\sum_{k} \int_{0}^{T}\left(\int_{\mathbb{T}^{2}} \nabla_{\xi} \mathbf{g}_{k}(\cdot, \mathbf{u})\left(\partial_{\gamma} \mathbf{u}, \partial_{\gamma} \mathbf{u}\right) \mathrm{d} x\right)^{2} \mathrm{~d} t\right]^{\frac{r}{4}} \\
& \leq c \mathbb{E}\left[\left(\int_{0}^{T}\left(\int_{\mathbb{T}^{2}}\left|\partial_{\gamma} \mathbf{u}\right|^{2} \mathrm{~d} x\right)^{2} \mathrm{~d} t\right]^{\frac{r}{4}}\right. \\
& \leq \delta \mathbb{E}\left[\sup _{0 \leq t \leq T} \int_{\mathbb{T}^{2}}|\nabla \mathbf{u}|^{2} \mathrm{~d} x\right]^{\frac{r}{2}}+c(\delta) \mathbb{E}\left[\int_{0}^{T} \int_{\mathbb{T}^{2}}|\nabla \mathbf{u}|^{2} \mathrm{~d} x \mathrm{~d} t\right]^{\frac{r}{2}} \\
& \leq \delta \mathbb{E}\left[\sup _{0 \leq t \leq T} \int_{\mathbb{T}^{2}}|\nabla \mathbf{u}|^{2} \mathrm{~d} x\right]^{\frac{r}{2}}+c(\delta) \mathbb{E}\left[1+\left\|\mathbf{u}_{0}\right\|_{L_{x}^{2}}^{2}\right]^{\frac{r}{2}}
\end{aligned}
$$

using (2.7) in the last step. By similar arguments we gain

$$
\begin{aligned}
\mathbb{E}\left[\sup _{0 \leq t \leq T}\left|(I I)_{2}^{2}\right|\right]^{\frac{r}{2}} & \leq c \mathbb{E}\left[\int_{0}^{T}\left(\int_{\mathbb{T}^{2}} \partial_{\gamma} \mathbf{g}_{k}(\cdot, \mathbf{u}) \cdot \partial_{\gamma} \mathbf{u} \mathrm{d} x\right)^{2} \mathrm{~d} t\right]^{\frac{r}{4}} \\
& \leq c \mathbb{E}\left[\left(\int_{0}^{T}\left(\int_{\mathbb{T}^{2}}\left|\partial_{\gamma} \mathbf{u}\right||\mathbf{u}| \mathrm{d} x\right)^{2} \mathrm{~d} t\right]^{\frac{r}{4}}\right. \\
& \leq c \mathbb{E}\left[\sup _{(0, T)} \int_{\mathbb{T}^{2}}|\mathbf{u}|^{2} \mathrm{~d} x+\int_{0}^{T} \int_{\mathbb{T}^{2}}|\nabla \mathbf{u}|^{2} \mathrm{~d} x \mathrm{~d} t\right]^{\frac{r}{2}} \\
& \leq c \mathbb{E}\left[1+\left\|\mathbf{u}_{0}\right\|_{L_{x}^{2}}^{2}\right]^{\frac{r}{2}} .
\end{aligned}
$$

Finally, we have by (2.1)

$$
\begin{aligned}
(I I I) & =\frac{1}{2} \int_{\mathbb{T}^{2}} \int_{0}^{t} \mathrm{~d}\left\langle\left\langle\int_{0} \partial_{\gamma}(\Phi(\mathbf{u}) \mathrm{d} W)\right\rangle \mathrm{d} x\right. \\
& =\frac{1}{2} \sum_{k} \int_{\mathbb{T}^{2}} \int_{0}^{t} \mathrm{~d}\left\langle\left\langle\int_{0} \partial_{\gamma}\left(\Phi(\mathbf{u}) e_{k}\right) \mathrm{d} \beta_{k}\right\rangle\right\rangle \mathrm{d} x \\
& \leq \frac{1}{2} \sum_{k} \int_{0}^{t} \int_{\mathbb{T}^{2}}\left|\nabla_{\xi} \mathbf{g}_{k}(\cdot, \mathbf{u}) \partial_{\gamma} \mathbf{u}\right|^{2} \mathrm{~d} x \mathrm{~d} \sigma \\
& +\frac{1}{2} \sum_{k} \int_{0}^{t} \int_{\mathbb{T}^{2}}\left|\partial_{\gamma} \mathbf{g}_{k}(\cdot, \mathbf{u})\right|^{2} \mathrm{~d} x \mathrm{~d} \sigma \\
& \leq c \int_{0}^{t} \int_{\mathbb{T}^{2}}|\nabla \mathbf{u}|^{2} \mathrm{~d} x \mathrm{~d} \sigma+c \int_{0}^{t} \int_{\mathbb{T}^{2}}|\mathbf{u}|^{2} \mathrm{~d} x \mathrm{~d} \sigma .
\end{aligned}
$$


Hence we obtain by (2.7) that

$$
\mathbb{E}\left[\sup _{0 \leq t \leq T}|(I I I)|\right]^{\frac{r}{2}} \leq c \mathbb{E}\left[1+\left\|\mathbf{u}_{0}\right\|_{L_{x}^{2}}^{2}\right]^{\frac{r}{2}}
$$

Plugging all together and choosing $\delta$ small enough we have shown (2.8).

The proof of (c) is similar: we simply differentiate once more. We apply Itô's formula to the function $f^{\beta}(\mathbf{u}):=\frac{1}{2}\left\|\partial^{\beta} \mathbf{u}\right\|_{L_{x}^{2}}^{2}$ where $\beta \in \mathbb{N}_{0}^{2}$ is a multi-index of length 2. We obtain

$$
\begin{aligned}
\frac{1}{2}\left\|\partial^{\beta} \mathbf{u}(t)\right\|_{L_{x}^{2}}^{2}= & \frac{1}{2}\left\|\partial^{\beta} \mathbf{u}_{0}\right\|_{L_{x}^{2}}^{2}+\int_{\mathbb{T}^{2}} \int_{0}^{t} \partial^{\beta} \mathbf{u} \cdot \mathrm{d} \partial^{\beta} \mathbf{u} \mathrm{d} x \\
& +\frac{1}{2} \int_{\mathbb{T}^{2}} \int_{0}^{t} \mathrm{~d}\left\|\int_{0}^{.} \partial^{\beta}(\Phi(\mathbf{u}) \mathrm{d} W)\right\| \mathrm{d} x=:(I)+(I I)+(I I I),
\end{aligned}
$$

where

$$
\begin{aligned}
(I I)^{\prime} & =-(I I)_{1}+(I I)_{2}-(I I)_{3}, \\
(I I)_{1} & :=\mu \int_{0}^{t} \int_{\mathbb{T}^{2}}\left|\partial^{\beta} \nabla \mathbf{u}\right|^{2} \mathrm{~d} x \mathrm{~d} \sigma, \\
(I I)_{2} & :=\int_{0}^{t} \int_{\mathbb{T}^{2}} \partial^{\beta} \mathbf{u} \cdot \partial^{\beta}(\Phi(\mathbf{u}) \mathrm{d} W) \mathrm{d} x, \\
(I I)_{3} & :=\int_{0}^{t} \int_{\mathbb{T}^{2}} \partial^{\beta}((\nabla \mathbf{u}) \mathbf{u}) \cdot \partial^{\beta} \mathbf{u} \mathrm{d} x \mathrm{~d} \sigma .
\end{aligned}
$$

The main difference is that $(I I)_{3}$ does not vanish. By [20, Lemma 2.1.20] (with $m=2$ ) and Young's inequality we have

$$
\begin{aligned}
\left|\int_{\mathbb{T}^{2}} \partial^{\beta} \operatorname{div}(\mathbf{u} \otimes \mathbf{u}) \cdot \partial^{\beta} \mathbf{u} \mathrm{d} x\right| & \leq c\|\mathbf{u}\|_{W_{x}^{3,2}}^{\frac{7}{4}}\|\mathbf{u}\|_{W_{x}^{1,2}}^{\frac{3}{4}}\|\mathbf{u}\|_{L_{x}^{2}}^{\frac{1}{2}} \\
& \leq \delta\|\mathbf{u}\|_{W_{x}^{3,2}}^{2}+c(\delta)\|\mathbf{u}\|_{W_{x}^{1,2}}^{6}\|\mathbf{u}\|_{L_{x}^{2}}^{4} \\
& \leq \delta\|\mathbf{u}\|_{W_{x}^{3,2}}^{2}+c(\delta)\left(\|\mathbf{u}\|_{W_{x}^{1,2}}^{10}+\|\mathbf{u}\|_{L_{x}^{2}}^{10}\right),
\end{aligned}
$$

where $\delta>0$ is arbitrary. This implies

$$
\begin{aligned}
\mathbb{E}\left[\sup _{0 \leq t \leq T}\left|(I I)_{3}\right|\right]^{\frac{r}{2}} & \leq \delta \mathbb{E}\left[\int_{0}^{T}\|\mathbf{u}\|_{W_{x}^{3,2}}^{2} \mathrm{~d} t\right]^{\frac{r}{2}}+c(\delta) \mathbb{E}\left[\sup _{0 \leq t \leq T}\|\mathbf{u}\|_{W_{x}^{1,2}}^{2}+\sup _{0 \leq t \leq T}\|\mathbf{u}\|_{L_{x}^{2}}^{2}\right]^{\frac{5 r}{2}} \\
& \leq \delta \mathbb{E}\left[\int_{0}^{T}\|\mathbf{u}\|_{W_{x}^{3,2}}^{2} \mathrm{~d} t\right]^{\frac{r}{2}}+c(\delta) \mathbb{E}\left[\left\|\mathbf{u}_{0}\right\|_{W_{x}^{1,2}}\right]^{\frac{5 r}{2}}
\end{aligned}
$$

due to (2.8). By arguments similar to the proof of (b), using (2.2), we gain

$$
\mathbb{E}\left[\sup _{0 \leq t \leq T}\left|(I I)_{2}\right|\right]^{\frac{r}{2}}
$$




$$
\begin{aligned}
& \leq \delta \mathbb{E}\left[\sup _{0 \leq t \leq T}\|\mathbf{u}\|_{W_{x}^{2,2}}^{2} \mathrm{~d} x\right]^{\frac{r}{2}}+c(\delta) \mathbb{E}\left[\sup _{0 \leq t \leq T}\|\mathbf{u}\|_{L_{x}^{2}}^{2}+\sup _{0 \leq t \leq T}\|\mathbf{u}\|_{W_{x}^{1,2}}^{2}+\int_{0}^{T}\|\mathbf{u}\|_{W_{x}^{2,2}}^{2} \mathrm{~d} t\right]^{\frac{r}{2}} \\
& \leq \delta \mathbb{E}\left[\sup _{0 \leq t \leq T}\|\mathbf{u}\|_{W_{x}^{2,2}}^{2} \mathrm{~d} x\right]^{\frac{r}{2}}+c \mathbb{E}\left[1+\left\|\mathbf{u}_{0}\right\|_{W_{x}^{1,2}}^{2}\right]^{\frac{r}{2}}
\end{aligned}
$$

using again (2.8). Finally, we get (2.7) that

$$
\mathbb{E}\left[\sup _{0 \leq t \leq T}|(I I I)|\right]^{\frac{r}{2}} \leq c \mathbb{E}\left[1+\left\|\mathbf{u}_{0}\right\|_{W_{x}^{1,2}}^{2}\right]^{\frac{r}{2}}
$$

arguing again similarly to (b) and using (2.2). Again the claim follows by choosing $\delta$ small enough.

Under the assumptions of Lemma 2 (b) equation (1.1) is satisfied strongly in the analytical sense. That is we have

$$
\mathbf{u}(t)=\mathbf{u}(0)+\int_{0}^{t}\left[\mu \Delta \mathbf{u}-(\nabla \mathbf{u}) \mathbf{u}-\nabla \pi_{\mathrm{det}}\right] \mathrm{d} \sigma+\int_{0}^{t}\left[\Phi(\mathbf{u})+\Phi^{\pi}\right] \mathrm{d} W
$$

$\mathbb{P}$-a.s. for all $t \in[0, T]$, recall equation (3.5). In the following we analyze the regularity of the pressure components $\pi_{\text {det }}$ and $\Phi^{\pi}$. In the following the subscript $w^{*}$ denotes Bochner-measurability with respect to the weak*-topology.

Corollary 1 (a) Under the assumptions of Lemma 2 we have

$$
\begin{array}{r}
\pi_{\operatorname{det}} \in L^{\frac{r}{2}}\left(\Omega, L^{2}\left(0, T ; L^{2}\left(\mathbb{T}^{2}\right)\right),\right. \\
\Phi^{\pi} \in L^{r}\left(\Omega ; L_{w^{*}}^{\infty}\left(0, T ; L_{2}\left(\mathfrak{U} ; L^{2}\left(\mathbb{T}^{2}\right)\right)\right)\right) .
\end{array}
$$

(b) Under the assumptions of Lemma 2(b) we have

$$
\begin{array}{r}
\pi_{\mathrm{det}} \in L^{\frac{r}{2}}\left(\Omega, L^{2}\left(0, T ; W^{1,2}\left(\mathbb{T}^{2}\right)\right),\right. \\
\Phi^{\pi} \in L^{r}\left(\Omega ; L_{w^{*}}^{\infty}\left(0, T ; L_{2}\left(\mathfrak{U} ; W^{1,2}\left(\mathbb{T}^{2}\right)\right)\right)\right) .
\end{array}
$$

(c) Under the assumptions of Lemma 2 (c) we have

$$
\begin{array}{r}
\pi_{\mathrm{det}} \in L^{\frac{r}{2}}\left(\Omega, L^{2}\left(0, T ; W^{2,2}\left(\mathbb{T}^{2}\right)\right),\right. \\
\Phi^{\pi} \in L^{r}\left(\Omega ; L_{w^{*}}^{\infty}\left(0, T ; L_{2}\left(\mathfrak{U} ; W^{2,2}\left(\mathbb{T}^{2}\right)\right)\right)\right) .
\end{array}
$$

Proof The key tool is the continuity of $\Delta^{-1}$ (from $W^{k-2, p}\left(\mathbb{T}^{2}\right) \rightarrow W^{k, p}\left(\mathbb{T}^{2}\right.$ ) for all $k \in \mathbb{N}_{0}$ and $\left.1<p<\infty\right)$. So, the regularity transfers from $\mathbf{u} \otimes \mathbf{u}$ to $\pi_{\text {det }}$. We obtain by Ladyshenskaya's inequality

$$
\int_{0}^{T} \int_{\mathbb{T}^{2}}\left|\pi_{\mathrm{det}}\right|^{2} \mathrm{~d} x \mathrm{~d} t=\int_{0}^{T} \int_{\mathbb{T}^{2}}\left|\Delta^{-1} \operatorname{div} \operatorname{div}(\mathbf{u} \otimes \mathbf{u})\right|^{2} \mathrm{~d} x \mathrm{~d} t \leq c \int_{0}^{T} \int_{\mathbb{T}^{2}}|\mathbf{u}|^{4} \mathrm{~d} x \mathrm{~d} t
$$




$$
\leq c\|\mathbf{u}\|_{L_{t}^{\infty} L_{x}^{2}}^{2}\|\nabla \mathbf{u}\|_{L_{t}^{2} L_{x}^{2}}^{2} \leq c\left(\|\mathbf{u}\|_{L_{t}^{\infty} L_{x}^{2}}^{4}+\|\nabla \mathbf{u}\|_{L_{t}^{2} L_{x}^{2}}^{4}\right)
$$

such that

$$
\mathbb{E}\left[\int_{0}^{T} \int_{\mathbb{T}^{2}}\left|\pi_{\mathrm{det}}\right|^{2} \mathrm{~d} x \mathrm{~d} t\right]^{\frac{r}{4}} \leq c \mathbb{E}\left[\|\mathbf{u}\|_{L_{t}^{\infty} L_{x}^{2}}^{2}+\|\nabla \mathbf{u}\|_{L_{t}^{2} L_{x}^{2}}^{2}\right]^{\frac{r}{2}}<\infty
$$

using Lemma 2 (a). The estimates for (b) and (c) are very similar. We have to compare $\nabla \pi$ with $|\mathbf{u}||\nabla \mathbf{u}|$, where we have

$$
|\mathbf{u}||\nabla \mathbf{u}| \leq|\mathbf{u}|^{2}+|\nabla \mathbf{u}|^{2} \in L^{\frac{r}{2}}\left(\Omega ; L^{2}(Q)\right)
$$

under the assumptions of Lemma 2 (b). Similarly, $\nabla^{2} \pi$ behaves as $|\mathbf{u}|\left|\nabla^{2} \mathbf{u}\right|+|\nabla \mathbf{u}|^{2}$ which belongs to the same class since Lemma 2 (c) applies.

Now, we investigate the regularity of $\Phi^{\pi}$. We use continuity $\Delta^{-1}$ to obtain

$$
\begin{aligned}
\mathbb{E}\left[\sup _{0 \leq t \leq T}\left\|\Phi^{\pi}\right\|_{L_{2}\left(\mathfrak{U} ; L_{x}^{2}\right)}^{2}\right]^{\frac{r}{2}} & =\mathbb{E}\left[\sup _{0 \leq t \leq T}\left\|\nabla \Delta^{-1} \operatorname{div} \Phi(\mathbf{u})\right\|_{L_{2}\left(\mathfrak{U} ; L_{x}^{2}\right)}^{2}\right]^{\frac{r}{2}} \\
& =\mathbb{E}\left[\sup _{0 \leq t \leq T} \sum_{k \geq 1}\left\|\nabla \Delta^{-1} \operatorname{div} \mathbf{g}_{k}(\cdot, \mathbf{u})\right\|_{L_{x}^{2}}^{2}\right]^{\frac{r}{2}} \\
& \leq c \mathbb{E}\left[\sup _{0 \leq t \leq T} \sum_{k \geq 1}\left\|\mathbf{g}_{k}(\cdot, \mathbf{u})\right\|_{L_{x}^{2}}^{2}\right]^{\frac{r}{2}} \\
& \leq c \mathbb{E}\left[1+\sup _{0 \leq t \leq T}\|\mathbf{u}\|_{L_{x}^{2}}^{2}\right]^{\frac{r}{2}}<\infty
\end{aligned}
$$

using (2.1) and Lemma 2 (a). Similarly, we have

$$
\begin{aligned}
\mathbb{E}\left[\sup _{0 \leq t \leq T}\left\|\Phi^{\pi}\right\|_{L_{2}\left(\mathfrak{U} ; W_{x}^{1,2}\right)}^{2}\right]^{\frac{r}{2}} & \leq c \mathbb{E}\left[\sup _{0 \leq t \leq T} \sum_{k \geq 1}\left\|\mathbf{g}_{k}(\cdot, \mathbf{u})\right\|_{W_{x}^{1,2}}^{2}\right]^{\frac{r}{2}} \\
& \leq c \mathbb{E}\left[1+\sup _{0 \leq t \leq T}\|\mathbf{u}\|_{W_{x}^{1,2}}^{2}\right]^{\frac{r}{2}}<\infty
\end{aligned}
$$

using (2.1) and Lemma 2 (b), as well as

$$
\begin{aligned}
\mathbb{E}\left[\sup _{0 \leq t \leq T}\left\|\Phi^{\pi}\right\|_{L_{2}\left(\mathfrak{U} ; W_{x}^{2,2}\right)}^{2}\right]^{\frac{r}{2}} & \leq c \mathbb{E}\left[\sup _{0 \leq t \leq T} \sum_{k \geq 1}\left\|\mathbf{g}_{k}(\cdot, \mathbf{u})\right\|_{W_{x}^{2,2}}^{2} \mathrm{~d} t\right]^{\frac{r}{2}} \\
& \leq c \mathbb{E}\left[1+\sup _{0 \leq t \leq T}\|\mathbf{u}\|_{W_{x}^{2,2}}^{2}\right]^{\frac{r}{2}}<\infty
\end{aligned}
$$

by (2.2) and the Lemma 2 (c). 
Finally, we investigate the time-regularity of the velocity field.

Corollary 2 (a) Let the assumptions of Lemma 2 (b) be satisfied for some $r>2$. Then we have

$$
\mathbb{E}\left[\|\mathbf{u}\|_{C^{\alpha}\left([0, T] ; L_{x}^{2}\right)}\right]^{\frac{r}{2}}<\infty
$$

for all $\alpha<\frac{1}{2}$.

(b) Let the assumptions of Lemma 2 (c) be satisfied for some $r>2$. Then we have

$$
\mathbb{E}\left[\|\mathbf{u}\|_{C^{\alpha}\left([0, T] ; W_{x}^{1,2}\right)}\right]^{\frac{r}{2}}<\infty
$$

for all $\alpha<\frac{1}{2}$.

Proof We start with (a) and analyse each term in equation (2.11) separately. Lemma 2 (b) implies

$$
\int_{0}^{\cdot} \Delta \mathbf{u} \mathrm{d} \sigma \in L^{r}\left(\Omega ; W^{1,2}\left(0, T ; L^{2}\left(\mathbb{T}^{2}\right)\right)\right) .
$$

As seen in the proof of Corollary $1 \pi_{\operatorname{det}}$ and $\mathbf{u} \otimes \mathbf{u}$ have the same regularity. In particular, Corollary 1 (b) yields

$$
\int_{0}^{.}\left(\operatorname{div}(\mathbf{u} \otimes \mathbf{u})+\nabla \pi_{\operatorname{det}}\right) d \sigma \in L^{\frac{r}{2}}\left(\Omega ; W^{1,2}\left(0, T ; L^{2}\left(\mathbb{T}^{2}\right)\right)\right) .
$$

Finally, we have

$$
\int_{0}^{.} \Phi(\mathbf{u}) \mathrm{d} W \in L^{r}\left(\Omega ; C^{\alpha}\left([0, T] ; L^{2}\left(\mathbb{T}^{2}\right)\right)\right) .
$$

by combing Lemma 1 with (2.3). The same conclusion holds for $\Phi^{\pi}$ using Lemma 1 (a). Plugging all together and noting the embedding $W^{1,2}(0, T ; X) \hookrightarrow C^{\alpha}([0, T] ; X)$ for any separably Banach space $X$ the claim follows.

The proof of (b) follows along the same lines using the higher regularity from Lemma 2 (c), Corollary 1 (c) and (2.4).

\subsection{Discretization in space}

We work with a standard finite element set-up for incompressible fluid mechanics, see e.g. [7,15]. We denote by $\mathscr{T}_{h}$ a quasi-uniform subdivision of $\mathbb{T}^{2}$ into triangles of maximal diameter $h>0$. For $\mathcal{S} \subset \mathbb{R}^{2}$ and $\ell \in \mathbb{N}_{0}$ we denote by $\mathscr{P}_{\ell}(\mathcal{S})$ the polynomials on $\mathcal{S}$ of degree less than or equal to $\ell$. Moreover, we set $\mathscr{P}_{-1}(\mathcal{S}):=\{0\}$. Let us characterize the finite element spaces $V^{h}\left(\mathbb{T}^{2}\right)$ and $P^{h}\left(\mathbb{T}^{2}\right)$ as

$$
V^{h}\left(\mathbb{T}^{2}\right):=\left\{\mathbf{v}_{h} \in W^{1,2}\left(\mathbb{T}^{2}\right):\left.\mathbf{v}_{h}\right|_{\mathcal{S}} \in \mathscr{P}_{i}(\mathcal{S}) \forall \mathcal{S} \in \mathscr{T}_{h}\right\}
$$




$$
P^{h}\left(\mathbb{T}^{2}\right):=\left\{\pi_{h} \in L^{2}\left(\mathbb{T}^{2}\right):\left.\pi_{h}\right|_{\mathcal{S}} \in \mathscr{P}_{j}(\mathcal{S}) \forall \mathcal{S} \in \mathscr{T}_{h}\right\}
$$

We will assume that $i$ and $j$ are both natural to get (2.15) below (this is different from [9], where also $j=0$ is allowed). In order to guarantee stability of our approximations we relate $V^{h}\left(\mathbb{T}^{2}\right)$ and $P^{h}\left(\mathbb{T}^{2}\right)$ by the inf-sup condition, that is we assume that

$$
\sup _{\mathbf{v}_{h} \in V^{h}\left(\mathbb{T}^{2}\right), \mathbf{v}_{h} \neq 0} \frac{\int_{\mathbb{T}^{2}} \operatorname{div} \mathbf{v}_{h} \pi_{h} \mathrm{~d} x}{\left\|\nabla \mathbf{v}_{h}\right\|_{L_{x}^{2}}} \geq C\left\|\pi_{h}\right\|_{L_{x}^{2}} \quad \forall \pi_{h} \in P^{h}\left(\mathbb{T}^{2}\right),
$$

where $C>0$ does not depend on $h$. This gives a relation between $i$ and $j$ (for instance the choice $(i, j)=(1,0)$ is excluded whereas $(i, j)=(2,0)$ is allowed $)$. Finally, we define the space of discretely solenoidal finite element functions by

$$
V_{\mathrm{div}}^{h}\left(\mathbb{T}^{2}\right):=\left\{\mathbf{v}_{h} \in V^{h}\left(\mathbb{T}^{2}\right): \int_{\mathbb{T}^{2}} \operatorname{div} \mathbf{v}_{h} \pi_{h} \mathrm{~d} x=0 \forall \pi_{h} \in P^{h}\left(\mathbb{T}^{2}\right)\right\}
$$

Let $\Pi_{h}: L^{2}\left(\mathbb{T}^{2}\right) \rightarrow V_{\text {div }}^{h}\left(\mathbb{T}^{2}\right)$ be the $L^{2}\left(\mathbb{T}^{2}\right)$-orthogonal projection onto $V_{\text {div }}^{h}\left(\mathbb{T}^{2}\right)$. The following results concerning the approximability of $\Pi_{h}$ are well-known (see, for instance [18]). There is $c>0$ independent of $h$ such that we have

$$
\int_{\mathbb{T}^{2}}\left|\frac{\mathbf{v}-\Pi_{h} \mathbf{v}}{h}\right|^{2} \mathrm{~d} x+\int_{\mathbb{T}^{2}}\left|\nabla \mathbf{v}-\nabla \Pi_{h} \mathbf{v}\right|^{2} \mathrm{~d} x \leq c \int_{\mathbb{T}^{2}}|\nabla \mathbf{v}|^{2} \mathrm{~d} x
$$

for all $\mathbf{v} \in W_{\operatorname{div}}^{1,2}\left(\mathbb{T}^{2}\right)$ and

$$
\int_{\mathbb{T}^{2}}\left|\frac{\mathbf{v}-\Pi_{h} \mathbf{v}}{h}\right|^{2} \mathrm{~d} x+\int_{\mathbb{T}^{2}}\left|\nabla \mathbf{v}-\nabla \Pi_{h} \mathbf{v}\right|^{2} \mathrm{~d} x \leq c h^{2} \int_{\mathbb{T}^{2}}\left|\nabla^{2} \mathbf{v}\right|^{2} \mathrm{~d} x
$$

for all $\mathbf{v} \in W_{\text {div }}^{2,2}\left(\mathbb{T}^{2}\right)$. Similarly, if $\Pi_{h}^{\pi}: L^{2}\left(\mathbb{T}^{2}\right) \rightarrow P^{h}\left(\mathbb{T}^{2}\right)$ denotes the $L^{2}\left(\mathbb{T}^{2}\right)$ orthogonal projection onto $P^{h}\left(\mathbb{T}^{2}\right)$, we have

$$
\int_{\mathbb{T}^{2}}\left|\frac{p-\Pi_{h}^{\pi} p}{h}\right|^{2} \mathrm{~d} x \leq c \int_{\mathbb{T}^{2}}|\nabla p|^{2} \mathrm{~d} x
$$

for all $p \in W^{1,2}\left(\mathbb{T}^{2}\right)$ and

$$
\int_{\mathbb{T}^{2}}\left|\frac{p-\Pi_{h}^{\pi} p}{h}\right|^{2} \mathrm{~d} x \leq c h^{2} \int_{\mathbb{T}^{2}}\left|\nabla^{2} p\right|^{2} \mathrm{~d} x
$$

for all $p \in W^{2,2}\left(\mathbb{T}^{2}\right)$. Note that (2.17) requires the assumption $j \geq 1$ in the definition of $P^{h}\left(\mathbb{T}^{2}\right)$, whereas (2.16) also holds for $j=0$. 


\section{Time-discretization}

We consider an equidistant partition of $[0, T]$ with mesh size $\Delta t=T / M$ and set $t_{m}=m \Delta t$. Let $\mathbf{u}_{0}$ be an $\mathfrak{F}_{0}$-mesureable random variable with values in $W_{\text {div }}^{1,2}\left(\mathbb{T}^{2}\right)$. We aim at constructing iteratively a sequence of $\mathfrak{F}_{t_{m}}$-measurable random variables $\mathbf{u}_{m}$ with values in $W_{\text {div }}^{1,2}\left(\mathbb{T}^{2}\right)$ such that for every $\phi \in W_{\text {div }}^{1,2}\left(\mathbb{T}^{2}\right)$ it holds true $\mathbb{P}$-a.s.

$$
\begin{gathered}
\int_{\mathbb{T}^{2}} \mathbf{u}_{m} \cdot \boldsymbol{\varphi} \mathrm{d} x+\Delta t\left(\int_{\mathbb{T}^{2}}\left(\nabla \mathbf{u}_{m}\right) \mathbf{u}_{m} \cdot \boldsymbol{\phi} \mathrm{d} x+\mu \int_{\mathbb{T}^{2}} \nabla \mathbf{u}_{m}: \nabla \boldsymbol{\phi} \mathrm{d} x\right) \\
=\int_{\mathbb{T}^{2}} \mathbf{u}_{m-1} \cdot \boldsymbol{\varphi} \mathrm{d} x+\int_{\mathbb{T}^{2}} \Phi\left(\mathbf{u}_{m-1}\right) \Delta_{m} W \cdot \boldsymbol{\varphi} \mathrm{d} x
\end{gathered}
$$

where $\Delta_{m} W=W\left(t_{m}\right)-W\left(t_{m-1}\right)$. The existence of a unique $\mathbf{u}_{m}$ (given $\mathbf{u}_{m-1}$ and $\Delta_{m} W$ ) solving (3.1) is straightforward as it is a stationary Navier-Stokes system. The following result follows from Lemma 3.1 in [9].

Lemma 3 Assume that $\mathbf{u}_{0} \in L^{2^{q}}\left(\Omega, W_{\text {div }}^{1,2}\left(\mathbb{T}^{2}\right)\right)$ for some $1 \leq q<\infty$. Suppose that $\Phi$ satisfies (2.1). Then the iterates $\left(\mathbf{u}_{m}\right)_{m=1}^{M}$ given by (3.1) are $\mathfrak{F}_{t_{m}}$-measurable. Moreover, the following estimates hold uniformly in $M$ :

$$
\begin{array}{r}
\mathbb{E}\left[\max _{1 \leq m \leq M}\left\|\mathbf{u}_{m}\right\|_{W_{x}^{1,2}}^{2^{q}}+\Delta t \sum_{k=1}^{M}\left\|\mathbf{u}_{m}\right\|_{W_{x}^{1,2}}^{2^{q}-2}\left\|\nabla^{2} \mathbf{u}_{m}\right\|_{L_{x}^{2}}^{2}\right] \leq c\left(q, T, \mathbf{u}_{0}\right), \\
\mathbb{E}\left[\sum_{k=1}^{M}\left\|\mathbf{u}_{m}-\mathbf{u}_{m-1}\right\|_{W_{x}^{1,2}}^{2}\left\|\nabla \mathbf{u}_{m}\right\|_{L_{x}^{2}}^{2}\right] \leq c\left(T, \mathbf{u}_{0}\right), \\
\mathbb{E}\left[\left(\sum_{k=1}^{M}\left\|\mathbf{u}_{m}-\mathbf{u}_{m-1}\right\|_{W_{x}^{1,2}}^{2}\right)^{4}+\left(\Delta t \sum_{k=1}^{M}\left\|\nabla^{2} \mathbf{u}_{m}\right\|_{L_{x}^{2}}^{2}\right)^{4}\right] \leq c\left(T, \mathbf{u}_{0}\right) .
\end{array}
$$

Now, for $\boldsymbol{\phi} \in W^{1,2}\left(\mathbb{T}^{2}\right)$ we can insert $\boldsymbol{\phi}-\nabla \Delta^{-1} \operatorname{div} \boldsymbol{\phi} \in W_{\text {div }}^{1,2}\left(\mathbb{T}^{2}\right)$ in (3.1) and obtain

$$
\begin{aligned}
\int_{\mathbb{T}^{2}} \mathbf{u}_{m} \cdot \boldsymbol{\varphi} \mathrm{d} x+\Delta t\left(\int_{\mathbb{T}^{2}}\left(\nabla \mathbf{u}_{m}\right) \mathbf{u}_{m} \cdot \boldsymbol{\phi} \mathrm{d} x+\mu \int_{\mathbb{T}^{2}} \nabla \mathbf{u}_{m}: \nabla \boldsymbol{\phi} \mathrm{d} x\right) \\
=\int_{\mathbb{T}^{2}} \mathbf{u}_{m-1} \cdot \boldsymbol{\varphi} \mathrm{d} x+\Delta t \int_{\mathbb{T}^{2}} \pi_{m}^{\mathrm{det}} \operatorname{div} \boldsymbol{\phi} \mathrm{d} x \\
\quad+\int_{\mathbb{T}^{2}} \Phi\left(\mathbf{u}_{m-1}\right) \Delta_{m} W \cdot \boldsymbol{\varphi} \mathrm{d} x+\int_{\mathbb{T}^{2}} \int_{0}^{t} \Phi_{m-1}^{\pi} \Delta_{m} W \cdot \boldsymbol{\varphi} \mathrm{d} x,
\end{aligned}
$$

where

$$
\begin{aligned}
\pi_{m}^{\mathrm{det}} & =-\Delta^{-1} \operatorname{div} \operatorname{div}\left(\mathbf{u}_{m} \otimes \mathbf{u}_{m}\right), \\
\Phi_{m-1}^{\pi} & =-\nabla \Delta^{-1} \operatorname{div} \Phi\left(\mathbf{u}_{m-1}\right) .
\end{aligned}
$$


Lemma 4 Assume that $\mathbf{u}_{0} \in L^{8}\left(\Omega, W_{\text {div }}^{1,2}\left(\mathbb{T}^{2}\right)\right)$ and that $\Phi$ satisfies $(2.1)$. For all $m \in\{1, \ldots, M\}$ the random variable $\pi_{m}^{\mathrm{det}}$ is $\mathfrak{F}_{t_{m}}$-measureable, has values in $W^{1,2}\left(\mathbb{T}^{2}\right)$ and we have

$$
\mathbb{E}\left[\Delta t \sum_{m=1}^{M}\left\|\nabla \pi_{m}^{\mathrm{det}}\right\|_{L_{x}^{2}}^{2}\right] \leq C
$$

uniformly in $\Delta t$.

Remark 1 A corresponding result is shown in [9, Lemma 3.2] for the full pressure provided the noise is divergence-free (in this case $\Phi_{m}^{\pi}$ vanishes). This is quite restrictive as it means that $\Phi$ is linear in $\mathbf{u}$.

Proof The $\mathfrak{F}_{t_{m}}$-measurability of $\pi_{m}^{\text {det }}$ follows directly from the one of $\mathbf{u}_{m}$ stated in Lemma 3. By continuity of the operator $\nabla \Delta^{-1} \operatorname{div}$ on $L^{2}\left(\mathbb{T}^{2}\right)$ we have

$$
\begin{aligned}
\left\|\nabla \pi_{m}^{\operatorname{det}}\right\|_{L_{x}^{2}}^{2} & \leq c\left\|\operatorname{div}\left(\mathbf{u}_{m} \otimes \mathbf{u}_{m}\right)\right\|_{L_{x}^{2}}^{2} \leq c\left\|\mathbf{u}_{m}\right\|_{L_{x}^{4}}^{2}\left\|\nabla \mathbf{u}_{m}\right\|_{L_{x}^{4}}^{2} \\
& \leq c\left\|\mathbf{u}_{m}\right\|_{L_{x}^{2}}\left\|\nabla \mathbf{u}_{m}\right\|_{L_{x}^{2}}^{2}\left\|\nabla^{2} \mathbf{u}_{m}\right\|_{L_{x}^{2}} \leq c\left\|\mathbf{u}_{m}\right\|_{L_{x}^{2}}^{2}+c\left\|\nabla \mathbf{u}_{m}\right\|_{L_{x}^{2}}^{4}\left\|\nabla^{2} \mathbf{u}_{m}\right\|_{L_{x}^{2}}^{2}
\end{aligned}
$$

$\mathbb{P}$-a.s. using also Ladyshenskaya's inequality $\|v\|_{L_{x}^{4}}^{2} \leq c\|v\|_{L_{x}^{2}}\|\nabla v\|_{L_{x}^{2}}$ which holds in two-dimensions. Now, summing with respect to $m$, applying expectations and using Lemma 3 yields the claim.

Lemma 5 Assume that $\mathbf{u}_{0} \in L^{8}\left(\Omega, W_{\text {div }}^{1,2}\left(\mathbb{T}^{2}\right)\right)$ and that $\Phi$ satisfies (2.1). For all $m \in\{1, \ldots, M\}$ the random variable $\Phi_{m}^{\pi}$ is $\mathfrak{F}_{t_{m}}$-measureable, has values in $L_{2}\left(\mathfrak{U} ; W^{1,2}\left(\mathbb{T}^{2}\right)\right)$ and we have

$$
\mathbb{E}\left[\Delta t \sum_{m=1}^{M}\left\|\Phi_{m}^{\pi}\right\|_{L_{2}\left(\mathfrak{U} ; W_{x}^{1,2}\right)}^{2}\right] \leq C
$$

uniformly in $\Delta t$.

Proof As for Lemma 4 the proof mainly relies on the continuity of $\nabla \Delta^{-1} \operatorname{div}$ on $L^{2}\left(\mathbb{T}^{2}\right)$. Here, we have by $(2.4)$

$$
\begin{aligned}
\left\|\Phi_{m}^{\pi}\right\|_{L_{2}\left(\mathfrak{U} ; W_{x}^{1,2}\right)}^{2} & =\sum_{k \geq 1}\left\|\nabla \Delta^{-1} \operatorname{div}\left(\Phi\left(\mathbf{u}_{m}\right) e_{k}\right)\right\|_{W_{x}^{1,2}}^{2} \\
& \leq c \sum_{k \geq 1}\left\|\Phi\left(\mathbf{u}_{m}\right) e_{k}\right\|_{W_{x}^{1,2}}^{2}=c\left\|\Phi\left(\mathbf{u}_{m}\right)\right\|_{L_{2}\left(\mathfrak{U} ; W_{x}^{1,2}\right)}^{2} \leq c\left(1+\left\|\nabla \mathbf{u}_{m}\right\|_{W_{x}^{1,2}}^{2}\right) .
\end{aligned}
$$

Summing over $m$, applying expectations and using Lemma 3 finishes the proof.

Following [9] we set for $\varepsilon>0$

$$
\Omega_{\Delta t}^{\varepsilon}=\left\{\omega \in \Omega \mid \max _{1 \leq m \leq M}\left\|\nabla \mathbf{u}_{m}\right\|_{L_{x}^{2}}^{2} \leq-\varepsilon \log (\Delta t)\right\}
$$


such that

$$
\mathbb{P}\left(\Omega_{\Delta t}^{\varepsilon}\right) \geq 1-\frac{\mathbb{E}\left[\max _{1 \leq m \leq M}\left\|\nabla \mathbf{u}_{m}\right\|_{L_{x}^{2}}^{2}\right]}{-\varepsilon \log (\Delta t)} \geq 1+\frac{C}{\varepsilon \log \Delta t}
$$

using Lemma 3. We obtain the following result.

Theorem 2 Assume that (2.1) holds and that $\mathbf{u}_{0} \in L^{8}\left(\Omega, W_{\text {div }}^{1,2}\left(\mathbb{T}^{2}\right)\right)$ is an $\mathfrak{F}_{0^{-}}$ measureable random variable. Let $\mathbf{u}$ be the unique strong solution to (1.1) in the sense of Definition 1. Assume that we have

$$
\mathbb{E}\left[\|\mathbf{u}\|_{C^{\alpha}\left([0, T] ; L_{x}^{4}\right)}^{4}\right]<\infty, \quad \mathbb{E}\left[\|\mathbf{u}\|_{C^{\alpha}\left([0, T] ; W_{x}^{1,2}\right)}^{2}\right]<\infty
$$

for some $\alpha \in\left(0, \frac{1}{2}\right)$; recall Corollary 2. Let $\left(\mathbf{u}_{m}\right)_{m=1}^{M}$ be the solution to (3.1). Then we have the error estimate

$$
\begin{aligned}
& \mathbb{E}\left[\mathbf { 1 } _ { \Omega _ { \Delta t } ^ { \varepsilon } } \left(\max _{1 \leq m \leq M}\left\|\mathbf{u}\left(t_{m}\right)-\mathbf{u}_{m}\right\|_{L_{x}^{2}}^{2}\right.\right.\left.\left.+\Delta t \sum_{m=1}^{M}\left\|\nabla \mathbf{u}\left(t_{m}\right)-\nabla \mathbf{u}_{m}\right\|_{L_{x}^{2}}^{2}\right)\right] \\
& \leq c_{\varepsilon}(\Delta t)^{2 \alpha-\varepsilon}
\end{aligned}
$$

for any $\varepsilon>0$.

Remark 2 - Estimate (4.3) improves the result from [9, Thm. 3.1], where the convergence rate is only $\alpha-\varepsilon$.

- In the paper [4] the time-discretization of the stochastic Navier-Stokes equations is analysed. The corresponding error does not contain an indicator function such as $\mathbf{1}_{\Omega_{\Delta t}^{\varepsilon}}$. The convergence rate is, however, only of logarithmic order.

Proof Subtracting (1.1) and (3.1) we obtain the equation for the error $\mathbf{e}_{m}=\mathbf{u}\left(t_{m}\right)-\mathbf{u}_{m}$ which reads as

$$
\begin{aligned}
& \int_{\mathbb{T}^{2}} \mathbf{e}_{m} \cdot \boldsymbol{\varphi} \mathrm{d} x+\int_{t_{m-1}}^{t_{m}}\left(\int_{\mathbb{T}^{2}}\left((\nabla \mathbf{u}) \mathbf{u}-\left(\nabla \mathbf{u}_{m}\right) \mathbf{u}_{m}\right) \cdot \boldsymbol{\phi} \mathrm{d} x+\mu \int_{\mathbb{T}^{2}} \nabla \mathbf{e}_{m}: \nabla \boldsymbol{\phi} \mathrm{d} x\right) \mathrm{d} t \\
& =\int_{\mathbb{T}^{2}} \mathbf{e}_{m-1} \cdot \boldsymbol{\varphi} \mathrm{d} x+\mu \int_{t_{m-1}}^{t_{m}} \int_{\mathbb{T}^{2}}\left(\nabla \mathbf{u}\left(t_{m}\right)-\nabla \mathbf{u}(t)\right): \nabla \boldsymbol{\phi} \mathrm{d} x \mathrm{~d} t \\
& \quad+\int_{\mathbb{T}^{2}}\left(\int_{t_{m-1}}^{t_{m}} \Phi(\mathbf{u}) \mathrm{d} W-\Phi\left(\mathbf{u}_{m-1}\right) \Delta_{m} W\right) \cdot \boldsymbol{\varphi} \mathrm{d} x
\end{aligned}
$$

for all $\boldsymbol{\phi} \in W_{\operatorname{div}}^{1,2}\left(\mathbb{T}^{2}\right)$. Choosing $\boldsymbol{\phi}=\mathbf{e}_{m}$ implies

$$
\begin{aligned}
& \frac{1}{2} \int_{\mathbb{T}^{2}}\left|\mathbf{e}_{m}\right|^{2} \mathrm{~d} x+\frac{1}{2} \int_{\mathbb{T}^{2}}\left|\mathbf{e}_{m}-\mathbf{e}_{m-1}\right|^{2} \mathrm{~d} x+\mu \Delta t \int_{\mathbb{T}^{2}}\left|\nabla \mathbf{e}_{m}\right|^{2} \mathrm{~d} x \\
& \quad=\frac{1}{2} \int_{\mathbb{T}^{2}}\left|\mathbf{e}_{m-1}\right|^{2} \mathrm{~d} x-\mu \int_{t_{m-1}}^{t_{m}} \int_{\mathbb{T}^{2}}\left(\nabla \mathbf{u}\left(t_{m}\right)-\nabla \mathbf{u}(t)\right): \nabla \mathbf{e}_{m} \mathrm{~d} x \mathrm{~d} t
\end{aligned}
$$




$$
\begin{aligned}
& +\int_{t_{m-1}}^{t_{m}} \int_{\mathbb{T}^{2}}\left((\nabla \mathbf{u}) \mathbf{u}-\left(\nabla \mathbf{u}_{m}\right) \mathbf{u}_{m}\right) \cdot \mathbf{e}_{m} \mathrm{~d} x \mathrm{~d} t \\
& +\int_{\mathbb{T}^{2}}\left(\int_{t_{m-1}}^{t_{m}} \Phi(\mathbf{u}) \mathrm{d} W-\Phi\left(\mathbf{u}_{m-1}\right) \Delta_{m} W\right) \cdot \mathbf{e}_{m} \mathrm{~d} x .
\end{aligned}
$$

Iterating this equality and following the arguments in [9, proof of Thm. 3.1] yields

$$
\begin{gathered}
\mathbb{E}\left[\mathbf{1}_{\Omega_{\Delta t}^{\varepsilon}}\left(\max _{1 \leq m \leq M}\left\|\mathbf{u}\left(t_{m}\right)-\mathbf{u}_{m}\right\|_{L_{x}^{2}}^{2}+\Delta t \sum_{m=1}^{M}\left\|\nabla \mathbf{u}\left(t_{m}\right)-\nabla \mathbf{u}_{m}\right\|_{L_{x}^{2}}^{2}\right)\right] \\
\leq c(\Delta t)^{2 \alpha-\varepsilon}+c \mathbb{E}\left[\sum_{m=1}^{M} \int_{t_{m-1}}^{t_{m}} \int_{\mathbb{T}^{2}}\left|\nabla \mathbf{u}\left(t_{m}\right)-\nabla \mathbf{u}(t)\right|^{2} \mathrm{~d} x \mathrm{~d} t\right] .
\end{gathered}
$$

Note that only the first bound from (3.7) has been used for (3.10). The second bound from (3.7), not used in [9], allows us to estimates the remaining integral by

$$
\mathbb{E}\left[\sum_{m=1}^{M} \int_{t_{m-1}}^{t_{m}} \int_{\mathbb{T}^{2}}\left|\nabla \mathbf{u}\left(t_{m}\right)-\nabla \mathbf{u}(t)\right|^{2} \mathrm{~d} x \mathrm{~d} t\right] \leq c(\Delta t)^{2 \alpha}
$$

which finishes the proof.

\section{Finite element based space-time approximation}

Now we consider a fully practical scheme combining the implicite Euler scheme in time (as in the last section) with a finite element approximation in space. For a given $h>0$ let $\mathbf{u}_{h, 0}$ be an $\mathfrak{F}_{0}$-mesureable random variable with values in $V_{\text {div }}^{h}\left(\mathbb{T}^{2}\right.$ ) (for instance $\Pi_{h} \mathbf{u}_{0}$ ). We aim at constructing iteratively a sequence of random variables $\mathbf{u}_{h, m}$ with values in $V_{\text {div }}^{h}\left(\mathbb{T}^{2}\right)$ such that for every $\boldsymbol{\phi} \in V_{\text {div }}^{h}\left(\mathbb{T}^{2}\right)$ it holds true $\mathbb{P}$-a.s.

$$
\begin{aligned}
& \int_{\mathbb{T}^{2}} \mathbf{u}_{h, m} \cdot \boldsymbol{\varphi} \mathrm{d} x+\Delta t \int_{\mathbb{T}^{2}}\left(\left(\nabla \mathbf{u}_{h, m}\right) \mathbf{u}_{h, m-1}+\left(\operatorname{div} \mathbf{u}_{h, m-1}\right) \mathbf{u}_{h, m}\right) \cdot \boldsymbol{\phi} \mathrm{d} x \\
& \quad+\mu \Delta t \int_{\mathbb{T}^{2}} \nabla \mathbf{u}_{m}: \nabla \boldsymbol{\phi} \mathrm{d} x=\int_{\mathbb{T}^{2}} \mathbf{u}_{h, m-1} \cdot \boldsymbol{\varphi} \mathrm{d} x+\int_{\mathbb{T}^{2}} \Phi\left(\mathbf{u}_{h, m-1}\right) \Delta_{m} W \cdot \boldsymbol{\varphi} \mathrm{d} x,
\end{aligned}
$$

where $\Delta_{m} W=W\left(t_{m}\right)-W\left(t_{m-1}\right)$. We quote the following result concerning the existence of solutions $\mathbf{u}_{h, m}$ to (4.1) from [8, Lemma 3.1].

Lemma 6 Let $1 \leq q<\infty$. Assume that $\mathbf{u}_{h, 0} \in L^{2^{q}}\left(\Omega, V_{\text {div }}^{h}\left(\mathbb{T}^{2}\right)\right)$ is an $\mathfrak{F}_{0}$-mesureable random variable and that $\mathbb{E}\left[\left\|\mathbf{u}_{h, 0}\right\|_{L_{x}^{2}}^{2^{q}} \mid\right] \leq K$ uniformly in $h$ for some $K>0$. Suppose that $\Phi$ satisfies (2.1). Then the iterates $\left(\mathbf{u}_{h, m}\right)_{m=1}^{M}$ given by (4.1) are $\mathfrak{F}_{t_{m}}$-measurable. 
Moreover, the following estimate holds uniformly in $M$ and $h$ :

$$
\mathbb{E}\left[\max _{1 \leq m \leq M}\left\|\mathbf{u}_{h, m}\right\|_{L_{x}^{2}}^{2^{q}}+\Delta t \sum_{k=1}^{M}\left\|\mathbf{u}_{h, m}\right\|_{L_{x}^{2}}^{2^{q}-2}\left\|\nabla \mathbf{u}_{h, m}\right\|_{L_{x}^{2}}^{2}\right] \leq c(q, T, K) .
$$

\subsection{Error analysis}

In this subsection we establish convergence with rates of the above defined algorithm. We introduce for $\varepsilon>0$ the sample set

$$
\Omega_{h}^{\varepsilon}=\left\{\omega \in \Omega \mid \max _{0 \leq m \leq M}\left(\left\|\nabla \mathbf{u}_{m}\right\|_{L_{x}^{2}}^{4}+\left\|\mathbf{u}_{h, m}\right\|_{L_{x}^{2}}^{2}\right) \leq-\varepsilon \log h\right\}
$$

which can be controlled by

$$
\mathbb{P}\left(\Omega_{h}^{\varepsilon}\right) \geq 1-\frac{\mathbb{E}\left[\max _{1 \leq m \leq M}\left(\left\|\nabla \mathbf{u}_{m}\right\|_{L_{x}^{2}}^{4}+\left\|\mathbf{u}_{h, m}\right\|_{L_{x}^{2}}^{2}\right)\right]}{-\varepsilon \log h} \geq 1+\frac{C}{\varepsilon \log h}
$$

using Lemma 3 and 6. We also recall the definition of $\Omega_{\Delta t}^{\varepsilon}$ in (3.6). We are now ready to state our main result.

Theorem 3 Let $\mathbf{u}_{0} \in L^{2}\left(\Omega, W_{\text {div }}^{1,2}\left(\mathbb{T}^{2}\right)\right) \cap L^{8}\left(\Omega ; L_{\text {div }}^{2}\left(\mathbb{T}^{2}\right)\right)$ be $\mathfrak{F}_{0}$-measurable and assume that $\Phi$ satisfies (2.1). Let $\mathbf{u}$ be the unique strong solution to (1.1) in the sense of Definition 1. Suppose further that

$$
\mathbb{E}\left[\|\mathbf{u}\|_{C^{\alpha}\left([0, T] ; L_{x}^{4}\right)}^{4}\right]<\infty, \quad \mathbb{E}\left[\|\mathbf{u}\|_{C^{\alpha}\left([0, T] ; W_{x}^{1,2}\right)}^{2}\right]<\infty
$$

for $\alpha \in\left(0, \frac{1}{2}\right)$; recall Corollary 2. Assume that $L \Delta t \leq(-\varepsilon \log h)^{-1}$ for some $L>0$. Then we have

$$
\begin{aligned}
& \mathbb{E}\left[\mathbf{1}_{\Omega_{\Delta t}^{\varepsilon} \cap \Omega_{h}^{\varepsilon}}\left(\max _{1 \leq m \leq M}\left\|\mathbf{u}\left(t_{m}\right)-\mathbf{u}_{h, m}\right\|_{L_{x}^{2}}^{2}+\sum_{m=1}^{M} \Delta t\left\|\nabla \mathbf{u}\left(t_{m}\right)-\nabla \mathbf{u}_{h, m}\right\|_{L_{x}^{2}}^{2}\right)\right] \\
& \leq c\left(h^{2}+(\Delta t)^{2 \alpha-\varepsilon}\right),
\end{aligned}
$$

where $\left(\mathbf{u}_{h, m}\right)_{m=1}^{M}$ is the solution to (4.1) with $\mathbf{u}_{h, 0}=\Pi_{h} \mathbf{u}_{0}$. The constant $c$ in (4.3) is independent of $M$ and $h$.

Remark 3 We do not expect that it is possible to avoid an indicator function in general (see [24] for the numerical approximation of stochastic PDEs with non-Lipschitz nonlinearities). But it is not clear if our choice of the sample subset is optimal.

The rest of the paper is devoted to the proof of Theorem 3. In fact Theorem 3 will follow from combining Theorem 2 with the following theorem which estimates the error between the time-discrete solution $\mathbf{u}_{m}$ to (3.1) and the solution $\mathbf{u}_{h, m}$ to (4.1). 
Theorem 4 Let $\mathbf{u}_{0} \in L^{2}\left(\Omega ; W_{\text {div }}^{1,2}\left(\mathbb{T}^{2}\right)\right) \cap L^{8}\left(\Omega ; L_{\text {div }}^{2}\left(\mathbb{T}^{2}\right)\right)$ be $\mathfrak{F}_{0}$-measurable and assume that $\Phi$ satisfies (2.1). Let $\left(\mathbf{u}_{m}\right)_{m=1}^{M}$ be the solution to (3.1). Assume that $L \Delta t \leq$ $(-\varepsilon \log h)^{-1}$ for some $L>0$. Then we have

$$
\begin{aligned}
& \mathbb{E}\left[\mathbf{1}_{\Omega_{h}^{\varepsilon}}\left(\max _{1 \leq m \leq M}\left\|\mathbf{u}_{m}-\mathbf{u}_{h, m}\right\|_{L_{x}^{2}}^{2}+\Delta t \sum_{m=1}^{M}\left\|\nabla \mathbf{u}_{m}-\nabla \mathbf{u}_{h, m}\right\|_{L_{x}^{2}}^{2}\right)\right] \\
& \quad \leq c\left(h^{2}+\Delta t\right)
\end{aligned}
$$

where $\left(\mathbf{u}_{h, m}\right)_{m=1}^{M}$ is the solution to (4.1) with $\mathbf{u}_{h, 0}=\Pi_{h} \mathbf{u}_{0}$. The constant $c$ is (4.4) is independent of $M$ and $h$.

Remark 4 In the estimate of [9, Thm. 4.1] the error is estimated by $h^{-3 \varepsilon}\left(h^{2}+(\Delta t)+\right.$ $\left.\frac{h^{2}}{\Delta t}\right)$. By our refined pressure analysis we are able to get rid of the term $\frac{h^{2}}{\Delta t}$ which leads to a restrictive assumption between space and time-discretization. Additionally, we can remove the factor $h^{-3 \varepsilon}$ arising from the convective term, see (4.6) below. Note that the error term $\frac{h^{2}}{\Delta t}$ also appears in [10], where the finite-element based space-time discretization of the stochastic Stokes equations (that is the linearized version of (1.1) without convective term) is studied.

Proof of Theorem 4 Define the error $\mathbf{e}_{h, m}=\mathbf{u}_{m}-\mathbf{u}_{h, m}$. Subtracting (3.5) and (4.1)we obtain

$$
\begin{aligned}
& \int_{\mathbb{T}^{2}} \mathbf{e}_{h, m} \cdot \boldsymbol{\varphi} \mathrm{d} x+\Delta t \int_{\mathbb{T}^{2}} \mu\left(\nabla \mathbf{u}_{m}-\nabla \mathbf{u}_{h, m}\right): \nabla \boldsymbol{\phi} \mathrm{d} x \\
& =\int_{\mathbb{T}^{2}} \mathbf{e}_{h, m-1} \cdot \boldsymbol{\varphi} \mathrm{d} x-\Delta t \int_{\mathbb{T}^{2}}\left(\left(\nabla \mathbf{u}_{m}\right) \mathbf{u}_{m}-\left(\left(\nabla \mathbf{u}_{h, m}\right) \mathbf{u}_{h, m-1}+\left(\operatorname{div} \mathbf{u}_{h, m-1}\right) \mathbf{u}_{h, m}\right)\right) \cdot \boldsymbol{\phi} \mathrm{d} x \\
& \quad+\int_{\mathbb{T}^{2}}\left(\Phi\left(\mathbf{u}_{m}\right)-\Phi\left(\mathbf{u}_{h, m-1}\right)\right) \Delta_{m} W \cdot \boldsymbol{\varphi} \mathrm{d} x \\
& \quad-\int_{\mathbb{T}^{2}} \nabla \Delta^{-1} \operatorname{div} \Phi\left(\mathbf{u}_{m-1}\right) \Delta_{m} W \cdot \boldsymbol{\varphi} \mathrm{d} x+\Delta t \int_{\mathbb{T}^{2}} \pi_{m}^{\operatorname{det}} \operatorname{div} \boldsymbol{\phi} \mathrm{d} x
\end{aligned}
$$

for every $\boldsymbol{\phi} \in V_{\text {div }}^{h}\left(\mathbb{T}^{2}\right)$. Setting $\boldsymbol{\phi}=\Pi_{h} \mathbf{e}_{h, m}$ and applying the identity $\mathbf{a} \cdot(\mathbf{a}-\mathbf{b})=$ $\frac{1}{2}\left(|\mathbf{a}|^{2}-|\mathbf{b}|^{2}+|\mathbf{a}-\mathbf{b}|^{2}\right.$ ) (which holds for any $\mathbf{a}, \mathbf{b} \in \mathbb{R}^{n}$ ) we gain

$$
\begin{aligned}
\int_{\mathbb{T}^{2}} & \frac{1}{2}\left(\left|\Pi_{h} \mathbf{e}_{h, m}\right|^{2}-\left|\Pi_{h} \mathbf{e}_{h, m-1}\right|^{2}+\left|\Pi_{h} \mathbf{e}_{h, m}-\Pi_{h} \mathbf{e}_{h, m-1}\right|^{2}\right) \mathrm{d} x+\Delta t \int_{\mathbb{T}^{2}} \mu\left|\nabla \mathbf{e}_{h, m}\right|^{2} \mathrm{~d} x \\
= & \Delta t \int_{\mathbb{T}^{2}} \mu \nabla \mathbf{e}_{h, m}: \nabla\left(\mathbf{u}_{m}-\Pi_{h} \mathbf{u}_{m}\right) \mathrm{d} x \\
& -\Delta t \int_{\mathbb{T}^{2}}\left(\left(\nabla \mathbf{u}_{m}\right) \mathbf{u}_{m}-\left(\left(\nabla \mathbf{u}_{h, m}\right) \mathbf{u}_{h, m-1}+\left(\operatorname{div} \mathbf{u}_{h, m-1}\right) \mathbf{u}_{h, m}\right)\right) \cdot \Pi_{h} \mathbf{e}_{h, m} \mathrm{~d} x \\
& +\Delta t \int_{\mathbb{T}^{2}} \pi_{m}^{\operatorname{det}} \operatorname{div} \Pi_{h} \mathbf{e}_{h, m} \mathrm{~d} x \\
& +\int_{\mathbb{T}^{2}}\left(\Phi\left(\mathbf{u}_{m}\right)-\Phi\left(\mathbf{u}_{h, m-1}\right)\right) \Delta_{m} W \cdot \Pi_{h} \mathbf{e}_{h, m} \mathrm{~d} x \\
& -\int_{\mathbb{T}^{2}} \nabla \Delta^{-1} \operatorname{div} \Phi\left(\mathbf{u}_{m-1}\right) \Delta_{m} W \cdot \Pi_{h} \mathbf{e}_{h, m} \mathrm{~d} x
\end{aligned}
$$




$$
=I_{1}(m)+\cdots+I_{5}(m) .
$$

Young's inequality yields

$$
\begin{aligned}
I_{1}(m) & \leq \kappa \Delta t \int_{\mathbb{T}^{2}}\left|\nabla \mathbf{e}_{h, m}\right|^{2} \mathrm{~d} x+c_{\kappa} \Delta t \int_{\mathbb{T}^{2}}\left|\nabla \mathbf{u}_{m}-\nabla \Pi_{h} \nabla \mathbf{u}_{m}\right|^{2} \mathrm{~d} x \\
& \leq \kappa \Delta t \int_{\mathbb{T}^{2}}\left|\nabla \mathbf{e}_{h, m}\right|^{2} \mathrm{~d} x+c_{\kappa} h^{2} \Delta t \int_{\mathbb{T}^{2}}\left|\nabla^{2} \mathbf{u}_{m}\right|^{2} \mathrm{~d} x .
\end{aligned}
$$

for every $\kappa>0$ using also (2.15). The convective term $I_{2}(m)$ can be decomposed as

$$
\begin{aligned}
I_{2}(m)= & I_{2}^{1}(m)+I_{2}^{2}(m)+I_{2}^{3}(m), \\
I_{2}^{1}(m)= & -\Delta t \int_{\mathbb{T}^{2}}\left(\nabla \mathbf{e}_{h, m}\right) \mathbf{u}_{m-1} \cdot\left(\mathbf{u}_{m}-\Pi_{h} \mathbf{u}_{m}\right) \mathrm{d} x, \\
I_{2}^{2}(m)= & \Delta t \int_{\mathbb{T}^{2}}\left(\nabla \mathbf{e}_{h, m}\right) \mathbf{e}_{h, m-1} \cdot\left(\mathbf{u}_{m}-\Pi_{h} \mathbf{u}_{m}\right) \mathrm{d} x \\
& +\Delta t \int_{\mathbb{T}^{2}}\left(\operatorname{div} \mathbf{e}_{h, m-1}\right) \mathbf{e}_{h, m} \cdot\left(\mathbf{u}_{m}-\Pi_{h} \mathbf{u}_{m}\right) \mathrm{d} x, \\
I_{2}^{3}(m)= & -\Delta t \int_{\mathbb{T}^{2}}\left(\nabla \Pi_{h} \mathbf{e}_{h, m}\right) \mathbf{e}_{h, m-1} \cdot \mathbf{u}_{m} \mathrm{~d} x \\
& -\Delta t \int_{\mathbb{T}^{2}}\left(\operatorname{div} \mathbf{e}_{h, m-1}\right) \Pi_{h} \mathbf{e}_{h, m} \cdot \mathbf{u}_{m} \mathrm{~d} x .
\end{aligned}
$$

As in [9, pages 2489-2491] we introduce the sample set

$$
\Omega_{h, m}^{\varepsilon}=\left\{\omega \in \Omega \mid \max _{0 \leq n \leq m}\left(\left\|\nabla \mathbf{u}_{n}\right\|_{L_{x}^{2}}^{4}+\left\|\mathbf{u}_{h, n}\right\|_{L_{x}^{2}}^{2}\right) \leq-\varepsilon \log h\right\}
$$

and obtain

$$
\begin{aligned}
\mathbf{1}_{\Omega_{h, m-1}^{\varepsilon}} I_{2}^{2}(m) \leq & \kappa \mathbf{1}_{\Omega_{h, m-1}^{\varepsilon}}\left(\left\|\nabla \mathbf{e}_{h, m-1}\right\|_{L_{x}^{2}}^{2}+\left\|\nabla \mathbf{e}_{h, m}\right\|_{L_{x}^{2}}^{2}\right) \\
& +c_{\kappa} \Delta t h^{4}\left(\max _{1 \leq n \leq m}\left\|\mathbf{e}_{h, n}\right\|_{L_{x}^{2}}^{2}\right)\left\|\nabla \mathbf{u}_{m}\right\|_{L_{x}^{2}}^{2}\left\|\nabla^{2} \mathbf{u}_{m}\right\|_{L_{x}^{2}}^{2}, \\
\mathbf{1}_{\Omega_{h, m-1}^{\varepsilon} I_{2}^{3}(m) \leq} & \kappa \mathbf{1}_{\Omega_{h, m-1}^{\varepsilon}}\left(\left\|\nabla \mathbf{e}_{h, m-1}\right\|_{L_{x}^{2}}^{2}+\left\|\nabla \mathbf{e}_{h, m}\right\|_{L_{x}^{2}}^{2}\right) \\
& +c_{\kappa} \Delta t \log \left(h^{-\varepsilon}\right)\left(\max _{1 \leq n \leq m} \mathbf{1}_{\Omega_{h, n-1}^{\varepsilon}}\left\|\mathbf{e}_{h, n}\right\|_{L_{x}^{2}}^{2}\right) \\
& +c_{\kappa} \Delta t\left\|\nabla\left(\mathbf{u}_{m}-\mathbf{u}_{m-1}\right)\right\|_{L_{x}^{2}}^{2}\left(\max _{1 \leq n \leq m}\left\|\nabla \mathbf{u}_{n}\right\|_{L_{x}^{2}}^{2}\right)\left(\max _{0 \leq n \leq m}\left\|\mathbf{e}_{h, n}\right\|_{L_{x}^{2}}^{2}\right),
\end{aligned}
$$

where $\kappa>0$ is arbitrary. For $I_{2}^{1}(m)$, however, we get a slightly better estimate than in [9] since our definition of $I_{2}^{1}(m)$ makes use of $\operatorname{div} \mathbf{u}^{m}=0$. We obtain

$$
\mathbf{1}_{\Omega_{h, m-1}^{\varepsilon}} I_{2}^{1}(m) \leq \kappa \mathbf{1}_{\Omega_{h, m-1}^{\varepsilon}}\left\|\nabla \mathbf{e}_{h, m}\right\|_{L_{x}^{2}}^{2}+c_{\kappa} h^{3} \log \left(h^{-\varepsilon}\right)\left\|\nabla^{2} \mathbf{u}_{m}\right\|_{L_{x}^{2}}^{2}
$$


due to (2.14).

The crucial point in this proof (making the essential difference to [9]) are the estimates for the pressure terms $I_{3}(m)$ and $I_{5}(m)$. We will estimate $I_{3}(m)$ first whereas $I_{5}(m)$ will only be bounded after iterating (4.5), see the estimates for $\mathscr{M}_{m, 2}$ below. By (2.15) we have

$$
\begin{aligned}
I_{3}(m) & =\Delta t \int_{\mathbb{T}^{2}}\left(\pi_{m}^{\mathrm{det}}-\Pi_{h}^{\pi} \pi_{m}^{\mathrm{det}}\right) \operatorname{div} \Pi_{h} \mathbf{e}_{h, m} \mathrm{~d} x \\
& \leq c_{\kappa} \Delta t \int_{\mathbb{T}^{2}}\left|\pi_{m}^{\mathrm{det}}-\Pi_{h}^{\pi} \pi_{m}^{\operatorname{det}}\right|^{2} \mathrm{~d} x+\kappa \Delta t \int_{\mathbb{T}^{2}}\left|\nabla \Pi_{h} \mathbf{e}_{h, m}\right|^{2} \mathrm{~d} x \\
& \leq c_{\kappa} \Delta t h^{2} \int_{\mathbb{T}^{2}}\left|\nabla \pi_{m}^{\operatorname{det}}\right|^{2} \mathrm{~d} x+\kappa \Delta t \int_{\mathbb{T}^{2}}\left|\nabla \mathbf{e}_{h, m}\right|^{2} \mathrm{~d} x,
\end{aligned}
$$

where $\kappa>0$ is arbitrary. Plugging all together and choosing $\kappa$ small enough (to absorb the corresponding terms to the left-hand side) we have shown

$$
\begin{aligned}
& \mathbf{1}_{\Omega_{h, m-1}^{\varepsilon}}\left(\int_{\mathbb{T}^{2}}\left|\Pi_{h} \mathbf{e}_{h, m}\right|^{2} \mathrm{~d} x+\int_{\mathbb{T}^{2}}\left|\Pi_{h} \mathbf{e}_{h, m}-\Pi_{h} \mathbf{e}_{h, m-1}\right|^{2} \mathrm{~d} x+\Delta t \int_{\mathbb{T}^{2}}\left|\nabla \mathbf{e}_{h, m}\right|^{2} \mathrm{~d} x\right) \\
& \leq \mathbf{1}_{\Omega_{h, m-1}^{\varepsilon}} \int_{\mathbb{T}^{2}}\left|\mathbf{e}_{h, m-1}\right|^{2} \mathrm{~d} x+c \Delta t \log \left(h^{-\varepsilon}\right)\left(\max _{1 \leq n \leq m} \mathbf{1}_{\Omega_{h, n-1}^{\varepsilon}}\left\|\mathbf{e}_{h, n}\right\|_{L_{x}^{2}}\right) \\
& +c \Delta t h^{2}\left(\int_{\mathbb{T}^{2}}\left|\nabla \pi_{m}^{\operatorname{det}}\right|^{2} \mathrm{~d} x+\int_{\mathbb{T}^{2}}\left(1+\left|\nabla \mathbf{u}_{m}\right|^{2}\right) \mathrm{d} x \int_{\mathbb{T}^{2}}\left|\nabla^{2} \mathbf{u}_{m}\right|^{2} \mathrm{~d} x\right) \\
& \quad+c \Delta t h^{4}\left(\max _{1 \leq n \leq m}\left\|\mathbf{e}_{h, n}\right\|_{L_{x}^{2}}^{2}\right) \int_{\mathbb{T}^{2}}\left|\nabla \mathbf{u}_{m}\right|^{2} \mathrm{~d} x \int_{\mathbb{T}^{2}}\left|\nabla^{2} \mathbf{u}_{m}\right|^{2} \mathrm{~d} x \\
& +c \Delta t \int_{\mathbb{T}^{2}}\left|\nabla\left(\mathbf{u}_{m}-\mathbf{u}_{m-1}\right)\right|^{2} \mathrm{~d} x\left(\max _{1 \leq n \leq m} \int_{\mathbb{T}^{2}}\left|\nabla \mathbf{u}_{n}\right|^{2} \mathrm{~d} x\right)\left(\max _{1 \leq n \leq m} \int_{\mathbb{T}^{2}}\left|\mathbf{e}_{h, n}\right|^{2} \mathrm{~d} x\right) \\
& \left.+c \mathbf{1}_{\Omega_{h, m-1}^{\varepsilon}} \int_{\mathbb{T}^{2}}\left(\int_{t_{m-1}}^{t_{m}} \Phi\left(\mathbf{u}_{m-1}\right)-\Phi\left(\mathbf{u}_{h, m-1}\right)\right) \mathrm{d} W\right) \cdot \Pi_{h} \mathbf{e}_{h, m} \mathrm{~d} x \\
& \quad-c \mathbf{1}_{\Omega_{h, m-1}^{\varepsilon}} \int_{\mathbb{T}^{2}}\left(\int_{t_{m-1}}^{t_{m}} \nabla \Delta^{-1} \operatorname{div} \Phi\left(\mathbf{u}_{m-1}\right) \mathrm{d} W\right) \cdot \Pi_{h} \mathbf{e}_{h, m} \mathrm{~d} x .
\end{aligned}
$$

Iterating this inequality yields

$$
\begin{aligned}
& \mathbf{1}_{\Omega_{h, m-1}^{\varepsilon}} \int_{\mathbb{T}^{2}}\left|\Pi_{h} \mathbf{e}_{h, m}\right|^{2} \mathrm{~d} x+\sum_{n=1}^{m} \mathbf{1}_{\Omega_{h, n-1}^{\varepsilon}}\left(\int_{\mathbb{T}^{2}}\left|\Pi_{h} \mathbf{e}_{h, n}-\Pi_{h} \mathbf{e}_{h, n-1}\right|^{2} \mathrm{~d} x+\Delta t \int_{\mathbb{T}^{2}}\left|\nabla \mathbf{e}_{h, n}\right|^{2} \mathrm{~d} x\right) \\
& \leq \int_{\mathbb{T}^{2}}\left|\mathbf{e}_{h, 0}\right|^{2} \mathrm{~d} x+c \Delta t \log \left(h^{-\varepsilon}\right) \sum_{n=1}^{m}\left(\max _{1 \leq \ell \leq n} \mathbf{1}_{\Omega_{h, \ell-1}^{\varepsilon}}\left\|\mathbf{e}_{h, \ell}\right\|_{L_{x}^{2}}^{2}\right) \\
& \quad+c h^{2} \Delta t \sum_{n=1}^{m}\left(\int_{\mathbb{T}^{2}}\left|\nabla \pi_{n}^{\operatorname{det}}\right|^{2} \mathrm{~d} x+\int_{\mathbb{T}^{2}}\left(1+\left|\nabla \mathbf{u}_{n}\right|^{2}\right) \mathrm{d} x \int_{\mathbb{T}^{2}}\left|\nabla^{2} \mathbf{u}_{n}\right|^{2} \mathrm{~d} x\right) \\
& \quad+c \Delta t h^{4} \sum_{n=1}^{m}\left(\max _{1 \leq \ell \leq n}\left\|\mathbf{e}_{h, \ell}\right\|_{L_{x}^{2}}^{2}\right) \int_{\mathbb{T}^{2}}\left|\nabla \mathbf{u}_{n}\right|^{2} \mathrm{~d} x \int_{\mathbb{T}^{2}}\left|\nabla^{2} \mathbf{u}_{n}\right|^{2} \mathrm{~d} x
\end{aligned}
$$




$$
\begin{aligned}
& +c \Delta t \sum_{n=1}^{m} \int_{\mathbb{T}^{2}}\left|\nabla\left(\mathbf{u}_{n}-\mathbf{u}_{n-1}\right)\right|^{2} \mathrm{~d} x\left(\max _{1 \leq \ell \leq n} \int_{\mathbb{T}^{2}}\left|\nabla \mathbf{u}_{\ell}\right|^{2} \mathrm{~d} x\right)\left(\max _{1 \leq \ell \leq n} \int_{\mathbb{T}^{2}}\left|\mathbf{e}_{h, \ell}\right|^{2} \mathrm{~d} x\right) \\
& \left.+c \sum_{n=1}^{m} \mathbf{1}_{\Omega_{h, n-1}^{\varepsilon}} \int_{\mathbb{T}^{2}}\left(\int_{t_{n-1}}^{t_{n}} \Phi\left(\mathbf{u}_{n-1}\right)-\Phi\left(\mathbf{u}_{h, n-1}\right)\right) \mathrm{d} W\right) \cdot \Pi_{h} \mathbf{e}_{h, n} \mathrm{~d} x \\
& -c \sum_{n=1}^{m} \mathbf{1}_{\Omega_{h, n-1}^{\varepsilon}} \int_{\mathbb{T}^{2}}\left(\int_{t_{n-1}}^{t_{n}} \nabla \Delta^{-1} \operatorname{div} \Phi\left(\mathbf{u}_{n-1}\right) \mathrm{d} W\right) \cdot \Pi_{h} \mathbf{e}_{h, n} \mathrm{~d} x .
\end{aligned}
$$

Now, we explain how to bound line by line in expectation. The error in the initial datum can be bounded by $h^{2}$ by (2.14) and the assumption $\mathbf{u}_{0} \in L^{2}\left(\Omega ; W_{\text {div }}^{1,2}\left(\mathbb{T}^{2}\right)\right)$. The second term on the right-hand side can be handled by the discrete Gronwall lemma using the assumption $L \Delta t \leq(-\varepsilon \log h)^{-1}$. The expectation of the second line is bounded by $h^{2}$ using Lemmas 3 and 4 (the estimate for $\nabla \pi_{m}^{\text {det }}$ is the first main ingredient in our proof). This estimate is better than the corresponding one in [9] as only the deterministic part of the pressure appears here. The expectation of the terms in the third line is bounded by

$$
\begin{aligned}
\mathbb{E} & {\left[\left(\max _{1 \leq \ell \leq M}\left\|\mathbf{e}_{h, \ell}\right\|_{L_{x}^{2}}^{2}\right)\left(\max _{1 \leq \ell \leq M}\left\|\nabla \mathbf{u}_{\ell}\right\|_{L_{x}^{2}}^{2}\right) \Delta t \sum_{n=1}^{M} \int_{\mathbb{T}^{2}}\left|\nabla^{2} \mathbf{u}_{n}\right|^{2} \mathrm{~d} x\right] } \\
& \leq\left(\mathbb{E}\left[\max _{1 \leq \ell \leq M}\left\|\mathbf{e}_{h, \ell}\right\|_{L_{x}^{2}}^{2}\right]^{2}\right)^{\frac{1}{2}}\left(\mathbb{E}\left[\max _{1 \leq \ell \leq M}\left\|\nabla \mathbf{u}_{\ell}\right\|_{L_{x}^{2}}^{2}\right]^{4}\right)^{\frac{1}{4}}\left(\mathbb{E}\left[\Delta t \sum_{n=1}^{M} \int_{\mathbb{T}^{2}}\left|\nabla^{2} \mathbf{u}_{n}\right|^{2} \mathrm{~d} x\right]^{4}\right)^{\frac{1}{4}} .
\end{aligned}
$$

This is uniformly bounded as a consequence of Lemmas 3 and 6 . The fourth line is controlled by $\Delta t$ due to the estimate

$$
\begin{aligned}
& \mathbb{E}\left[\sum_{n=1}^{m} \int_{\mathbb{T}^{2}}\left|\nabla\left(\mathbf{u}_{n}-\mathbf{u}_{n-1}\right)\right|^{2} \mathrm{~d} x\left(\max _{1 \leq \ell \leq n} \int_{\mathbb{T}^{2}}\left|\nabla \mathbf{u}_{\ell}\right|^{2} \mathrm{~d} x\right)\left(\max _{1 \leq \ell \leq n} \int_{\mathbb{T}^{2}}\left|\mathbf{e}_{h, \ell}\right|^{2} \mathrm{~d} x\right)\right] \\
& \leq\left(\mathbb{E}\left[\sum_{n=1}^{M} \int_{\mathbb{T}^{2}}\left|\nabla\left(\mathbf{u}_{n}-\mathbf{u}_{n-1}\right)\right|^{2} \mathrm{~d} x\right]^{2}\right)^{\frac{1}{2}}\left(\mathbb{E}\left[\max _{1 \leq \ell \leq M} \int_{\mathbb{T}^{2}}\left|\nabla \mathbf{u}_{\ell}\right|^{2} \mathrm{~d} x\right]^{4}\right)^{\frac{1}{4}} \times \\
& \times\left(\mathbb{E}\left[\max _{1 \leq \ell \leq M} \int_{\mathbb{T}^{2}}\left|\mathbf{e}_{h, \ell}\right|^{2} \mathrm{~d} x\right]^{4}\right)^{\frac{1}{4}}
\end{aligned}
$$

and the uniform bounds from Lemmas 3 and 6 (with $q=3$ ). It remains to estimate the two stochastic terms

$$
\begin{aligned}
& \mathscr{M}_{m, 1}=\sum_{n=1}^{m} \mathbf{1}_{\Omega_{h, n-1}^{\varepsilon}} \int_{\mathbb{T}^{2}} \int_{t_{n-1}}^{t_{n}}\left(\Phi\left(\mathbf{u}_{n-1}\right)-\Phi\left(\mathbf{u}_{h, n-1}\right)\right) \mathrm{d} W \cdot \Pi_{h} \mathbf{e}_{h, n} \mathrm{~d} x \\
& \mathscr{M}_{m, 2}=\sum_{n=1}^{m} \mathbf{1}_{\Omega_{h, n-1}^{\varepsilon}} \int_{\mathbb{T}^{2}} \int_{t_{n-1}}^{t_{n}}\left(\mathrm{Id}-\Pi_{h}^{\pi}\right) \Delta^{-1} \operatorname{div} \Phi\left(\mathbf{u}_{n-1}\right) \mathrm{d} W \operatorname{div} \Pi_{h} \mathbf{e}_{h, n} \mathrm{~d} x .
\end{aligned}
$$


Note that we used integration by parts and $\Pi_{h} \mathbf{e}_{h, n} \in V_{\text {div }}^{h}\left(\mathbb{T}^{2}\right)$ together with the definition of $\Pi_{h}^{\pi}$ to rewrite $\mathscr{M}_{m, 2}$ into the form above. Finally, we write

$$
\begin{aligned}
\mathscr{M}_{m, 1}= & \sum_{n=1}^{m} \mathbf{1}_{\Omega_{h, n-1}^{\varepsilon}} \int_{\mathbb{T}^{2}} \int_{t_{n-1}}^{t_{n}}\left(\Phi\left(\mathbf{u}_{n-1}\right)-\Phi\left(\mathbf{u}_{h, n-1}\right)\right) \mathrm{d} W \cdot \Pi_{h} \mathbf{e}_{h, n-1} \mathrm{~d} x \\
& +\sum_{n=1}^{m} \mathbf{1}_{\Omega_{h, n-1}^{\varepsilon}} \int_{\mathbb{T}^{2}} \int_{t_{n-1}}^{t_{n}}\left(\Phi\left(\mathbf{u}_{n-1}\right)-\Phi\left(\mathbf{u}_{h, n-1}\right)\right) \mathrm{d} W \cdot\left(\Pi_{h} \mathbf{e}_{h, n}-\Pi_{h} \mathbf{e}_{h, n-1}\right) \mathrm{d} x \\
= & : \mathscr{M}_{m, 1}^{1}+\mathscr{M}_{m, 1}^{2}
\end{aligned}
$$

as well as

$$
\begin{aligned}
& \mathscr{M}_{m, 2}=\sum_{n=1}^{m} \mathbf{1}_{\Omega_{h, n-1}^{\varepsilon}} \int_{\mathbb{T}^{2}} \int_{t_{n-1}}^{t_{n}}\left(\operatorname{Id}-\Pi_{h}^{\pi}\right) \Delta^{-1} \operatorname{div} \Phi\left(\mathbf{u}_{n-1}\right) \mathrm{d} W \operatorname{div} \Pi_{h} \mathbf{e}_{h, n-1} \mathrm{~d} x \\
& +\sum_{n=1}^{m} \mathbf{1}_{\Omega_{h, n-1}^{\varepsilon}} \int_{\mathbb{T}^{2}} \int_{t_{n-1}}^{t_{n}}\left(\operatorname{Id}-\Pi_{h}^{\pi}\right) \Delta^{-1} \operatorname{div} \Phi\left(\mathbf{u}_{n-1}\right) \mathrm{d} W \operatorname{div}\left(\Pi_{h} \mathbf{e}_{h, n}-\Pi_{h} \mathbf{e}_{h, n-1}\right) \mathrm{d} x \\
& =: \mathscr{M}_{m, 2}^{1}+\mathscr{M}_{m, 2}^{2} .
\end{aligned}
$$

These representations have the advantage that $\mathscr{M}_{m, 1}^{1}$ and $\mathscr{M}_{m, 2}^{2}$ are martingales (note the index $n-1$ in the indicator functions). Consequently, we can apply the BurkholderDavis-Gundy inequality to estimate them. As far as $\mathscr{M}_{m, 1}$ is concerned we have

$$
\begin{aligned}
& \mathbb{E}\left[\max _{1 \leq m \leq M}\left|\mathscr{M}_{m, 1}^{1}\right|\right] \\
& \leq c \mathbb{E}\left[\sum_{n=1}^{M} \mathbf{1}_{\Omega_{h, n-1}^{\varepsilon}} \int_{t_{n-1}}^{t_{n}}\left\|\Phi\left(\mathbf{u}_{n-1}\right)-\Phi\left(\mathbf{u}_{h, n-1}\right)\right\|_{L_{2}\left(\mathfrak{U}, L_{x}^{2}\right)}^{2}\left\|\Pi_{h} \mathbf{e}_{h, n-1}\right\|_{L_{x}^{2}}^{2} \mathrm{~d} t\right]^{\frac{1}{2}} \\
& \leq c \mathbb{E}\left[\max _{0 \leq n \leq M} \mathbf{1}_{\Omega_{h, n}^{\varepsilon}}\left\|\Pi_{h} \mathbf{e}_{h, n}\right\|_{L_{x}^{2}}\left(\sum_{n=1}^{M} \mathbf{1}_{\Omega_{h, n-1}^{\varepsilon}} \int_{t_{n-1}}^{t_{n}}\left\|\Phi\left(\mathbf{u}_{n-1}\right)-\Phi\left(\mathbf{u}_{h, n-1}\right)\right\|_{L_{2}\left(\mathfrak{U}, L_{x}^{2}\right)}^{2} \mathrm{~d} t\right)^{\frac{1}{2}}\right] \\
& \leq \kappa \mathbb{E}\left[\max _{0 \leq n \leq M} \mathbf{1}_{\Omega_{h, n}^{\varepsilon}}\left\|\Pi_{h} \mathbf{e}_{h, n}\right\|_{L_{x}^{2}}^{2}\right]+c_{\kappa} \mathbb{E}\left[\sum_{n=1}^{M} \mathbf{1}_{\Omega_{h, n-1}^{\varepsilon}} \int_{t_{n-1}}^{t_{n}}\left\|\mathbf{u}_{n-1}-\mathbf{u}_{h, n-1}\right\|_{L_{x}^{2}}^{2} \mathrm{~d} t\right] \\
& \leq \kappa \mathbb{E}\left[\max _{0 \leq n \leq M} \mathbf{1}_{\Omega_{h, n}^{\varepsilon}}\left\|\Pi_{h} \mathbf{e}_{h, n}\right\|_{L_{x}^{2}}^{2}\right]+c_{\kappa} \mathbb{E}\left[\Delta t \sum_{n=1}^{M} \mathbf{1}_{\Omega_{h, n-1}^{\varepsilon}}\left\|\Pi_{h} \mathbf{e}_{h, n-1}\right\|_{L_{x}^{2}}^{2}\right] \\
& +c_{\kappa} \mathbb{E}\left[\Delta t \sum_{n=1}^{M} \mathbf{1}_{\Omega_{h, n-1}^{\varepsilon}}\left\|\mathbf{u}_{n-1}-\Pi_{h} \mathbf{u}_{n-1}\right\|_{L_{x}^{2}}^{2}\right]
\end{aligned}
$$

Here, we also used (2.1) as well as Young's inequality for $\kappa>0$ arbitrary. Now, the first term can be absorbed for $\kappa$ small enough. The second term can be handled by the discrete Gronwall lemma (note that $\Omega_{h, n}^{\varepsilon} \subset \Omega_{h, n-1}^{\varepsilon}$ such that $\mathbf{1}_{\Omega_{h, n}^{\varepsilon}} \leq \mathbf{1}_{\Omega_{h, n-1}^{\varepsilon}} \mathbb{P}$ a.s.) 
Using (2.14) the last term can be estimated by

$$
c_{\kappa} h^{2} \mathbb{E}\left[\Delta t \sum_{n=1}^{M} \mathbf{1}_{\Omega_{h, n-1}^{\varepsilon}}\left\|\nabla \mathbf{u}_{n-1}\right\|_{L_{x}^{2}}^{2}\right]
$$

which is bounded by $c h^{2}$ using Lemma 3 (recall that $\mathbf{u}_{0} \in L^{2}\left(\Omega ; W^{1,2}\left(\mathbb{T}^{2}\right)\right.$ )). For the term $\mathscr{M}_{m, 1}^{2}$ we obtain by Cauchy-Schwartz inequality, Young's inequality, Itôisometry and (2.1)

$$
\begin{aligned}
\mathbb{E}\left[\max _{1 \leq m \leq M}\left|\mathscr{M}_{m, 1}^{2}\right|\right] \leq & \kappa \mathbb{E}\left[\sum_{n=1}^{M} \mathbf{1}_{\Omega_{h, n-1}^{\varepsilon}}\left\|\Pi_{h} \mathbf{e}_{h, n}-\Pi_{h} \mathbf{e}_{h, n-1}\right\|_{L_{x}^{2}}^{2}\right] \\
& +c_{\kappa} \mathbb{E}\left[\sum_{n=1}^{M} \mathbf{1}_{\Omega_{h, n-1}^{\varepsilon}}\left\|\int_{t_{n-1}}^{t_{n}}\left(\Phi\left(\mathbf{u}_{n-1}\right)-\Phi\left(\mathbf{u}_{h, n-1}\right)\right) \mathrm{d} W\right\|_{L_{x}^{2}}^{2}\right] \\
\leq & \kappa \mathbb{E}\left[\sum_{n=1}^{M} \mathbf{1}_{\Omega_{h, n-1}^{\varepsilon}}\left\|\Pi_{h} \mathbf{e}_{h, n}-\Pi_{h} \mathbf{e}_{h, n-1}\right\|_{L_{x}^{2}}^{2}\right] \\
& +c_{\kappa} \mathbb{E}\left[\sum_{n=1}^{M} \mathbf{1}_{\Omega_{h, n-1}^{\varepsilon}} \int_{t_{n-1}}^{t_{n}}\left\|\mathbf{u}_{n-1}-\mathbf{u}_{h, n-1}\right\|_{L_{x}^{2}}^{2} \mathrm{~d} t\right]
\end{aligned}
$$

The first term can be absorbed for $\kappa$ small enough whereas the second one can be estimated as for $\mathscr{M}_{m, 1}^{1}$.

Now, we come to the second main ingredient which is the estimate for $\mathscr{M}_{m, 2}$. We obtain using (2.17), (2.1) and continuity of $\nabla^{2} \Delta^{-1}$

$$
\begin{aligned}
& \mathbb{E}\left[\max _{1 \leq m \leq M}\left|\mathscr{M}_{m, 2}^{1}\right|\right] \\
& \leq c \mathbb{E}\left[\sum_{n=1}^{M} \mathbf{1}_{\Omega_{h, n-1}} \int_{t_{n-1}}^{t_{n}}\left\|\left(\mathrm{Id}-\Pi_{h}^{\pi}\right) \Delta^{-1} \operatorname{div} \Phi\left(\mathbf{u}_{n-1}\right)\right\|_{L_{2}\left(\mathfrak{U}, L_{x}^{2}\right)}^{2}\left\|\nabla \Pi_{h} \mathbf{e}_{h, n-1}\right\|_{L_{x}^{2}}^{2} \mathrm{~d} t\right]^{\frac{1}{2}} \\
& \leq c h^{2} \mathbb{E}\left[\max _{1 \leq n \leq M}\left\|\nabla^{2} \Delta^{-1} \operatorname{div} \Phi\left(\mathbf{u}_{n-1}\right)\right\|_{L_{2}\left(\mathfrak{U}, L_{x}^{2}\right)}\left(\sum_{n=1}^{M} \mathbf{1}_{\Omega_{h, n-1}} \int_{t_{n-1}}^{t_{n}}\left\|\nabla \Pi_{h} \mathbf{e}_{h, n}\right\|_{L_{x}^{2}}^{2} \mathrm{~d} t\right)^{\frac{1}{2}}\right] \\
& \leq c_{\kappa} h^{4} \mathbb{E}\left[\max _{1 \leq n \leq M}\left\|\nabla \mathbf{u}_{n-1}\right\|_{L_{x}^{2}}^{2}\right]+\kappa \mathbb{E}\left[\Delta t \sum_{n=1}^{M} \mathbf{1}_{\Omega_{h, n-1}}\left\|\nabla \Pi_{h} \mathbf{e}_{h, n}\right\|_{L_{x}^{2}}^{2}\right] .
\end{aligned}
$$

The first term is bounded by $c_{\kappa} h^{4}$ using Lemmas 3 (recall that $\mathbf{u}_{0} \in L^{2}\left(\Omega ; W^{1,2}\left(\mathbb{T}^{2}\right)\right)$ ). The second term can be absorbed if $\kappa \ll 1$. As a consequence of Young's inequality, inverse estimates on $V^{h}\left(\mathbb{T}^{2}\right)$, Itô-ismotry and (2.17) we infer

$$
\mathbb{E}\left[\max _{1 \leq m \leq M}\left|\mathscr{M}_{m, 2}^{2}\right|\right] \leq \kappa h^{2} \mathbb{E}\left[\sum_{n=1}^{M} \mathbf{1}_{\Omega_{h, n-1}}\left\|\nabla\left(\Pi_{h} \mathbf{e}_{h, n}-\Pi_{h} \mathbf{e}_{h, n-1}\right)\right\|_{L_{x}^{2}}^{2}\right]
$$




$$
\begin{aligned}
& \quad+c_{\kappa} h^{-2} \mathbb{E}\left[\sum_{n=1}^{M} \mathbf{1}_{\Omega_{h, n-1}}\left\|\int_{t_{n-1}}^{t_{n}}\left(\mathrm{Id}-\Pi_{h}^{\pi}\right) \Delta^{-1} \operatorname{div} \Phi\left(\mathbf{u}_{n-1}\right) \mathrm{d} W\right\|_{L_{x}^{2}}^{2}\right] \\
& \leq c \kappa \mathbb{E}\left[\sum_{n=1}^{M} \mathbf{1}_{\Omega_{h, n-1}}\left\|\Pi_{h} \mathbf{e}_{h, n}-\Pi_{h} \mathbf{e}_{h, n-1}\right\|_{L_{x}^{2}}^{2}\right] \\
& +c_{\kappa} h^{-2} \mathbb{E}\left[\sum_{n=1}^{M} \mathbf{1}_{\Omega_{h, n-1}} \int_{t_{n-1}}^{t_{n}}\left\|\left(\mathrm{Id}-\Pi_{h}^{\pi}\right) \Delta^{-1} \operatorname{div} \Phi\left(\mathbf{u}_{n-1}\right)\right\|_{L_{2}\left(\mathfrak{U} ; L_{x}^{2}\right)}^{2} \mathrm{~d} t\right] \\
& \leq c \kappa \mathbb{E}\left[\sum_{n=1}^{M} \mathbf{1}_{\Omega_{h, n-1}}\left\|\Pi_{h} \mathbf{e}_{h, n}-\Pi_{h} \mathbf{e}_{h, n-1}\right\|_{L_{x}^{2}}^{2}\right] \\
& +c_{\kappa} h^{2} \mathbb{E}\left[\sum_{n=1}^{M} \int_{t_{n-1}}^{t_{n}}\left\|\nabla^{2} \Delta^{-1} \operatorname{div} \Phi\left(\mathbf{u}_{n-1}\right)\right\|_{L_{2}\left(\mathfrak{U} ; L_{x}^{2}\right)}^{2} \mathrm{~d} t\right] .
\end{aligned}
$$

The first term can be absorbed for $\kappa$ small enough. Arguing as for $\mathscr{M}_{m, 2}^{1}$ the last term is bounded by $c h^{2} \mathbb{E}\left[\max _{n}\left\|\nabla \mathbf{u}_{n-1}\right\|_{L_{x}^{2}}^{2}\right] \leq c h^{2}$. Plugging all together and noting that $\Omega_{h}^{\varepsilon} \subset \bigcup_{n=1}^{M} \Omega_{h, n}^{\varepsilon}$ shows

$$
\mathbb{E}\left[\mathbf{1}_{\Omega_{h}^{\varepsilon}}\left(\max _{1 \leq m \leq M}\left\|\Pi_{h} \mathbf{e}_{h, m}\right\|_{L_{x}^{2}}^{2}+\Delta t \sum_{m=1}^{M}\left\|\nabla \mathbf{e}_{h, m}\right\|_{L_{x}^{2}}^{2}\right)\right] \leq c\left(h^{2}+\Delta t\right) .
$$

Recalling that $\mathbf{e}_{h, m}=\mathbf{u}_{m}-\Pi_{h} \mathbf{u}_{m}+\Pi_{h} \mathbf{e}_{h, m}$ and using (2.14) as well as Lemma 3 (a) gives the claim.

Open Access This article is licensed under a Creative Commons Attribution 4.0 International License, which permits use, sharing, adaptation, distribution and reproduction in any medium or format, as long as you give appropriate credit to the original author(s) and the source, provide a link to the Creative Commons licence, and indicate if changes were made. The images or other third party material in this article are included in the article's Creative Commons licence, unless indicated otherwise in a credit line to the material. If material is not included in the article's Creative Commons licence and your intended use is not permitted by statutory regulation or exceeds the permitted use, you will need to obtain permission directly from the copyright holder. To view a copy of this licence, visit http://creativecommons.org/licenses/by/4.0/.

\section{References}

1. Bensoussan, A., Temam, R.: Équations stochastiques du type Navier-Stokes. J. Funct. Anal. 13, 195222 (1973)

2. Birnir, B.: The Kolmogorov-Obukhov statistical theory of turbulence. J. Nonlinear Sci. 23, 657-688 (2013)

3. Bessaih, H., Brzeźniak, Z., Millet, A.: Splitting up method for the 2D stochastic Navier-Stokes equations. Stoch. PDE Anal. Comput. 2, 433-470 (2014)

4. Bessaih, H., Millet, A.: Strong $L^{2}$ convergence of time numerical schemes for the stochastic twodimensional Navier-Stokes equations IMA. J. Num. Anal. 39, 2135-2167 (2019)

5. Breit, D.: Existence theory for stochastic power law fluids. J. Math. Fluid Mech. 17, 295-326 (2015)

6. Breit, D.: Existence Theory for Generalized Newtonian Fluids. Mathematics in Science and Engineering. Elsevier, Academic Press, London (2017) 
7. Brezzi, F., Fortin, M.: Mixed and Hybrid Finite Element Methods. Springer Series in Computational Mathematics, vol. 5. Springer, New York (1991)

8. Brzeźniak, Z., Carelli, E., Prohl, J.A.: Finite-element-based discretizations of the incompressible Navier-Stokes equations with multiplicative random forcing. IMA J. Num. Anal. 33, 771-824 (2013)

9. Carelli, E., Prohl, A.: Rates of convergence for discretizations of the stochastic incompressible NavierStokes equations. SIAM J. Numer. Anal. 50(5), 2467-2496 (2012)

10. Carelli, E., Hausenblas, E., Prohl, A.: Time-splitting methods to solve the stochastic incompressible Stokes equation. SIAM J. Numer. Anal. 50, 2917-2939 (2012)

11. Capiński, M.: A note on uniqueness of stochastic Navier-Stokes equations. Univ. Iagell. Acta Math. 30, 219-228 (1993)

12. Capiński, M., Cutland, N.J.: Stochastic Navier-Stokes equations. Acta Appl. Math. 25, 59-85 (1991)

13. Da Prato, G., Zabczyk, J.: Stochastic Equations in Infinite Dimensions. Encyclopedia of Mathematics and its Applications, vol. 44. Cambridge University Press, Cambridge (1992)

14. Flandoli, F., Gatarek, D.: Martingale and stationary solutions for stochastic Navier-Stokes equations. Probab. Theory Relat. Fields 102, 367-391 (1995)

15. Girault, V., Raviart, P.A.: Finite Element Method for Navier-Stokes Equations: Theory and Algorithms. Springer, Berlin, Heidelberg, New York (1981)

16. Girault, V., Lions, J.L.: Two-grid finite-element schemes for the steady Navier-Stokes problem in polyhedra. Port. Math. (N.S.) 58, 25-57 (2001)

17. Girault, V., Scott, L.R.: A quasi-local interpolation operator preserving the discrete divergence. Calcolo 40, 1-19 (2003)

18. Heywood, J.G., Rannacher, R.: Finite element approximation of the nonstationary Navier-Stokes problem. I. Regularity of solutions and second-order error estimates for spatial discretization. SIAM J. Numer. Anal. 19, 275-311 (1982)

19. Hofmanová, M.: Degenerate parabolic stochastic partial differential equations. Stoch. Process. Appl. 123(12), 4294-4336 (2013)

20. Kuksin, S., Shirikyan, A.: Mathematics of Two-Dimensional Turbulence, Volume 194 of Cambridge Tracts in Mathematics. Cambridge University Press, Cambridge (2012)

21. Mikulevicius, R., Rozovskii, B.L.: Stochastic Navier-Stokes equations for turbulent flows. SIAM J. Math. Anal. 35(5), 1250-1310 (2004)

22. Pardoux, E.: Equations aux dérivées Partielles stochastiques non linéaires monotones. Etude de solutions fortes de type Itô, Ph.d. thesis, Université Paris Sud (1975)

23. Prévôt, C., Röckner, M.: A concise course on stochastic partial differential equations. Lecture Notes in Mathematics, vol. 1905. Springer, Berlin (2007)

24. Printems, J.: On the discretization in time of parabolic stochastic partial differential equations. Math. Mod. Numer. Anal. 35, 1055-1078 (2001)

25. Romito, M.: Some probabilistic topics in the Navier-Stokes equations. Recent progress in the theory of the Euler and Navier-Stokes equations. London Mathematical Social Lecture Note Series, vol. 430, pp. 175-232. Cambridge University Press, Cambridge (2016)

26. Scott, L.R., Zhang, S.: Finite element interpolation of nonsmooth functions satisfying boundary conditions. Math. Comput. 54(190), 483-493 (1990)

27. Yan, Y.: Semidiscrete Galerkin approximation for a linear stochastic parabolic partial differential equation driven by an additive noise. Num. Math. 44, 829-847 (2004)

28. Yan, Y.: Galerkin finite element methods for stochastic parabolic partial differential equations. SIAM J. Numer. Anal. 43, 1363-1384 (2005)

Publisher's Note Springer Nature remains neutral with regard to jurisdictional claims in published maps and institutional affiliations. 